\title{
Particle and Parallel Momentum Balance Equations with Inclusion of Drifts, for Modelling Strong- to Weakly-Collisional Edge Plasmas
}

\author{
A V Chankin and P C Stangeby ${ }^{\mathrm{b}}$ \\ ${ }^{a}$ Max-Planck-Institut für Plasmaphysik, Boltzmannstraße 2, 85748 Garching, Germany \\ ${ }^{b}$ University of Toronto Institute for Aerospace Studies, 4925 Dufferin St., Toronto, \\ M3H 5T6, Canada
}

\begin{abstract}
A system of plasma particle and parallel momentum balance equations is derived appropriate for understanding the role of drifts in the edge and for edge modelling, particularly in the scrape-off layer (SOL) of tokamaks, stellarators and other magnetic confinement devices. The formulation allows for strong collisionality - but also covers the case of weak collisionality and strong drifts, a combination often encountered in the SOL. The most important terms are identified by assessing the magnitude of characteristic velocities and fluxes for the plasma edge region. Explanations of the physical nature of each term are provided. A number of terms that are sometimes not included in edge modelling, has been included in the parallel momentum balance equation after detailed analysis of the parallel component of the gradient of the total pressure-stress tensor. This includes terms related to curvature and divergence of the field lines, as well as further contributions coming from viscous forces related mainly to the ion centrifugal drift. All these terms are shown to be roughly of the same order of magnitude as convective momentum fluxes related to drifts, and therefore should be included in the momentum balance equation.
\end{abstract}




\section{Introduction}

Cross-field drift flows play an important role in influencing plasma parameters in the scrapeoff layer (SOL) and divertor of tokamaks [1]. They are believed to be responsible for changes in the in-out asymmetries caused by the reversal of the toroidal field direction, observed in many experiments, thereby affecting particle and power exhaust in the divertor/target (see e.g. [2-9]), including the tendency for the inner divertor to be detached for the 'standard' field direction (ion $\nabla$ B-drift toward the divertor in single-null configuration). The drifts are also believed to make a significant impact on the plasma parallel flows in the main SOL, far away from the divertor/target, where the influence of the interaction with neutrals on the plasma transport is not critical [10-15].

Attempts to quantitatively relate measured asymmetries and parallel flows to theoretical cross-field drifts using code-modelling have met with only limited success to date and it is not clear that all of the controlling physics has been identified and included in the codemodelling: some basic physical effect(s) other than drifts may also be playing an important role. The identification of any such additional effects requires that the theoretical cross-field drifts be fully and correctly described in the code-modelling. In the present paper the particle and momentum conservation equations are re-derived with the aim of more completely and correctly identifying the non-collisional drift terms, including the effects of curvature and divergence of the magnetic field. Fast parallel flows and divertor asymmetries appear to have a number of important practical implications for magnetic fusion devices, including the transfer of impurities such as carbon to the usually cooler inner divertor region, resulting in co-deposition trapping of tritium in a non-saturating process [16-21]. The motivation for improved understanding and modelling is therefore strong. Extensive theoretical and modelling effort in recent years have confirmed the significant role of drifts in both in-out asymmetries and parallel flows [22-39]. Ref. [39] however, presents a convincing demonstration that present-day $2 \mathrm{D}$ edge codes are failing to predict the large parallel ion flows widely measured in the SOL. Whether this is due to improper implementation of drifts into the codes, the result of poloidal variations in turbulent transport coefficients, or caused by other, unknown reasons, is at present unclear.

Proper accounting of the drift effects in theories and code modelling, however, has proven to be a difficult job, to a large extent because of the need for correct averaging of drift velocities of individual particles and their various products in order to obtain fluid equations for macroscopic plasma parameters. At the edge of the plasma, and in particular in the SOL, derivation of transport equations which include drifts is aggravated by difficulties associated with the presence of divertors/limiters that introduce poloidal asymmetries, large mean parallel velocity (especially in the SOL, with Mach numbers $~ 1$ ), and also because of the effect of neutrals on the plasma.

The usual practice in modelling of the edge, and in particular, the SOL plasma, is to assume anomalous perpendicular coefficients, sometimes poloidally dependent, for particle, heat and momentum transport, typically diffusion coefficients. This assumption is required in order to make the modelling results consistent with experimental observations where plasma turbulence is seen to determine perpendicular transport which greatly exceeds classical (and sometimes even neoclassical) transport. At the same time, drift fluxes, as already pointed out, play an important role in edge transport. In order to account for the most important contributions to the plasma transport, and for the sake of clarity of the analysis and the results, particle and parallel momentum balance equations in the present paper will be 
formulated in a way which clearly separates (a) guiding centre drifts, (b) parallel transport including parallel viscous forces, and (c) perpendicular anomalous particle and momentum fluxes. In addition, the effects of classical collisional perpendicular transport considered e.g. in [40] have to be added separately. They, as well as other collisional perpendicular transport, e.g. fast ion scattering, will not be evaluated in this paper.

Particle and parallel momentum balance equations for the edge plasma in the Pfirsch-Schlüter regime of frequent collisions that include drifts, adapted for the use in numerical codes, can be found in various sources (see e.g. [22,26,28,29,31,35]). References [26,31,35] describe the main equations used in numerical codes in magnetic configurations with poloidal divertors: EDGE2D, UEDGE and B2.5, respectively. The SOL of magnetically-confined plasmas is often only weakly collisional, while at the same time, drift effects can be strong. We have therefore in Sec. 2 employed a new method for 'opening' the total pressure-stress tensor which avoids the standard small perturbation analysis assumption of Chapman and Cowling, Braginskii, Spitzer, etc.

Here we will follow the practice adopted in the implementation of drifts in EDGE2D [26], namely, to formulate transport equations in such a way that clearly separates guiding centre drifts from Larmor rotation. In the most important terms describing perpendicular transport of both particles and momentum, contributions by the Larmor rotation, which numerically are the largest, can then be eliminated from the equations, as a result of a certain cancellation. This cancellation has been explicitly demonstrated for the particle equation [40] and is also claimed to hold for the momentum equation [41]. In a separate study we will explicitly demonstrate this cancellation for the momentum equation ([42], to be submitted for publication). This cancellation has proven to greatly improve the stability of numerical codes. The fluid parallel momentum balance equation is thus effectively reduced to the parallel momentum balance equation for guiding centres of Larmor orbits. The analysis performed in the present paper is more rigorous than that in Ref. [26] and we find a number of important terms that have not been included in this earlier work. Some of these terms can also be found in a later paper [35, as discussed in Sec. 7]. In this paper we analyse only particle and parallel momentum balance equations, leaving to future work the formulation of the complete set of particle, momentum and energy balance equations.

The general form of the particle and momentum plasma fluid equations is presented below:

$$
\left\{\begin{array}{l}
\frac{\partial n}{\partial t}+\vec{\nabla} \cdot(n \mathbf{V})=S \\
\frac{\partial\left(n m V_{\|}\right)}{\partial t}+(\vec{\nabla} \cdot \overrightarrow{\mathbf{P}})_{\|}-e Z n E_{\|}=R_{\|}+F_{\|}
\end{array}\right.
$$

This equation is applied to each plasma species. The notations are conventional and are explained in Sec. 2 where the total pressure-stress tensor, $\overrightarrow{\mathbf{P}}=n m\langle\mathbf{v v}\rangle$, in the fluid momentum conservation equation is 'opened', i.e. re-written in terms of the basic fluid variables, $n, \mathbf{V}, p_{\perp}$ and $p_{\|}$( or $T_{\perp}$ and $T_{\|}$) and/or their gradients, also involving terms specifying the divergence and curvature of the magnetic field. $\overrightarrow{\mathbf{P}}$ is opened directly, permitting direct insertion of classical drift velocities, $\mathbf{v}_{\perp, d r}$, which are calculated from individual particle motion given in Sec. 3. (Collisional perpendicular drift velocities, $\mathbf{v}_{\perp \text {,coll }}$, could also be inserted directly, but that is not done here.) The expression obtained in Sec. 2 
for the opened $\overrightarrow{\mathbf{P}}$ allows for arbitrary magnetic geometry and for arbitrary levels of collisionality. In Sec. 4 the expressions for $\mathbf{v}_{\perp, d r}$ taken from Sec. 3 are inserted into the opened expression for $\overrightarrow{\mathbf{P}}$. Here capital letter $\mathrm{V}$ indicates fluid velocity and small letter $\mathrm{v}$, individual particle velocity.

In Sec. 5 we focus attention on a particular drift-term resulting from opening $\overrightarrow{\mathbf{P}}$, namely $F_{\text {div }}=-\vec{\nabla} \cdot\left(n m\left\langle v_{\|} \mathbf{v}_{\perp}\right\rangle\right)$, which includes terms of the form $\left\langle\tilde{v}_{\|} \tilde{\mathbf{v}}_{\perp}\right\rangle$, the viscous terms. The random $\tilde{\mathbf{v}}_{\perp}$ that is involved here is not the Larmor velocity $\mathbf{v}_{\perp, L}$, but rather perpendicular drifts which include a random component due to a dependence of the drift speed on the magnitude of $\bar{v}_{\|}$and $\bar{v}_{\perp}$, where here the latter (with bar above $v$ ) indicate the velocities of the individual particle averaged over a Larmor orbit, see Eq. (24).

In Sec. 6 we address the critical issue of closure of the fluid equations, including approximations for the parallel viscous stress, $\Pi_{\|}$, which is often introduced as part of the closure process. Up until the point of closing the set of equations, the particle and momentum equations here are fully general as to the degree of collisionality. Closure, however, requires that a specific level of collisionality be assumed.

Finally, the summary of the results and comparison with earlier formulations are presented in Sec. 7.

\section{Opening the parallel component of the gradient of the total pressure-stress tensor}

The general form of the particle and momentum plasma fluid equations, Eq. (1), are obtained by assuming a distribution of particle velocities $f(t, \mathbf{r}, \mathbf{v})$ which is given by the Boltzmann kinetic equation. The Boltzmann equation is then multiplied by 1 and $m \mathbf{v}$ and integrated over individual particle velocity $\mathbf{v}$ to give Eq. (1) for ions: (see e.g. [40] (Eq. (1.12)), [43] (Eqs. (2.13) and (2.15))). Here $\mathbf{V}$ and $V_{\|}$are mean total (vector) and parallel fluid velocities, respectively, averaged over the distribution function, $S$ is the particle source, $\overrightarrow{\mathbf{P}}=n m\langle\mathbf{v v}\rangle$ is the total pressure-stress tensor, $\mathbf{v}$ is individual particle velocity, $\langle\ldots\rangle$ denotes averaging over the distribution function, $R_{\|}$is parallel friction force between ions and electrons and $F_{\|}$stands for all other forces (including forces arising from interaction with neutrals and external forces, as well as any momentum introduced in association with particle source $S$ ); other notations are conventional. There is a similar momentum equation for the electrons.

The objective in this paper is to 'open' $\overrightarrow{\mathbf{P}}=n m\langle\mathbf{v v}\rangle$ - specifically $(\vec{\nabla} \cdot \overrightarrow{\mathbf{P}})_{\|}$- i.e. to re-write it in a form where the expressions for perpendicular drift velocities - in particular the noncollisional ones - can be directly inserted, allowing for arbitrary levels of collisionality, and also allowing for arbitrary magnetic geometry. In order to accommodate arbitrary levels of collisionality, the ion momentum equation is formulated in terms of two pressures, parallel and perpendicular.

Different approaches have been employed to open $(\vec{\nabla} \cdot \overrightarrow{\mathbf{P}})_{\|}$, governed by the method chosen to close the set of fluid equations. The closure method is chosen on the basis of the degree of (ee, ii, ei) collisionality. Perhaps the best known approach is that of Chapman and Cowling, 
Spitzer, Braginskii [40], etc., which is appropriate for strong collisionality. In this approach the individual particle velocity is divided into two components - a mean velocity $\mathbf{V}$ and a random velocity $\tilde{\mathbf{v}}=\mathbf{v}-\mathbf{V}$, where $\mathrm{V}$ will be identified with the fluid velocity, thus $(\vec{\nabla} \cdot \overrightarrow{\mathbf{P}})_{\|}=(\vec{\nabla} \cdot(n m \mathbf{V} \mathbf{V}))_{\|}+(\vec{\nabla} \cdot \overrightarrow{\widetilde{\mathbf{P}}})_{\|}$, where $\stackrel{\widetilde{\mathbf{P}}}{=}=n m\langle\tilde{\mathbf{v}} \widetilde{\mathbf{V}}\rangle$ is the pressure-stress tensor (as distinct from $\overrightarrow{\mathbf{P}}$, the total pressure-stress tensor, which includes $\mathbf{V}$ ). $\overrightarrow{\widetilde{\mathbf{P}}}$ includes both ordinary static pressure and viscous terms. Consistent with the assumption of strong collisionality, a single isotropic pressure $p$ is assumed. The convective component is retained on the left hand side (LHS) of the equation and is combined with the time derivative to form the so-called substantive derivative, while the second term is moved to the right hand side (RHS). Consistent with the strong collisionality assumption, this approach employs a small perturbation analysis, starting from a Maxwellian distribution perturbed by the existence of gradients of density, temperature and velocity and with relaxation due to self-collisions. It is therefore appropriate that Braginskii etc. then define a viscosity tensor $\overrightarrow{\mathbf{\Pi}} \equiv \ddot{\widetilde{\mathbf{P}}}-p \overrightarrow{\mathbf{I}}$, where $\overrightarrow{\mathbf{I}}$ is the unit matrix and $p$ is the single, static, isotropic pressure, $p \equiv n m\left\langle\tilde{v}^{2}\right\rangle / 3$, since for the unperturbed (Maxwellian, possibly drifting, as occurs in the limit of infinitely strong collisionality) velocity distribution $\ddot{\widetilde{\mathbf{P}}}=p \overrightarrow{\mathbf{I}}$ and $\vec{\Pi}=0$, and so all of the perturbing effects are isolated in $\overrightarrow{\boldsymbol{\Pi}}=n m\left\langle\tilde{\mathbf{v}} \tilde{\mathbf{v}}-\overrightarrow{\mathbf{I}} \tilde{v}^{2} / 3\right\rangle$. (The general practice, following Braginskii, is to call $\vec{\Pi}$ the 'stress tensor', but to avoid confusion it will be called the 'viscosity tensor' here.) Braginskii etc. include a third fluid conservation equation - for energy - one each for electrons and ions, thus providing equations for $p_{e, i}$, but this does not close the set of equations since there are a number of quantities that still need to be related to the primary dependant variables $n, \mathbf{V}_{e, i}$ and $p_{e, i}$ given by the 5 fluid conservation equations, namely, some specific quantities that appear in the momentum equations, (a) $R_{/ / e i}\left(=-R_{/ / i e}\right.$ ), (b) $\vec{\Pi}_{e, i}$, as well as some specific quantities that appear in the energy equations, (c) $\mathbf{q}_{e, i}$, the heat flux density and (d) $Q_{e, i}$, heat gained by electrons and ions as a result of $e i$ collisions. From rudimentary kinetic theory one can roughly estimate all these latter quantities as transport coefficients, e.g. $q_{\|} \sim-n v_{t h} \lambda\left(d T / d s_{/ /}\right)$, which depends on collisionality through the mean free path $\lambda$, thus closing the equation set. For better estimates one can use the Chapman and Cowling, Braginskii, etc., method of employing small perturbation analysis, that is these quantities are related to gradients of the primary fluid variables $n, \mathbf{V}_{e, i}$ and $p_{e, i}$ (or $T_{e, i}$ ) using an expansion of the distribution function about a Maxwellian in the parameter $\lambda / L$, where $\lambda$ is the collisional mean free path and $L$ is the scale length of the gradients of $n, \mathbf{V}_{\mathrm{e}, \mathrm{i}}$ and $p_{e, i}$. This analysis is therefore valid in the limit of strong collisionality. (As an aside, one may note that by adding the electron and ion momentum (also energy) equations together, the need to estimate $R_{/ / e i}$ and $Q_{e, i}$ can be avoided since the $e$ and $i$ terms cancel.)

A completely different approach - and one which we will broadly follow here - was employed by Zawaideh, Najamabadi, and Conn, [44], which applies to the full range of collisionality, from strong to weak. In a strong magnetic field the anisotropy of the velocity distribution is not necessarily small, unless collisionality is very strong, and it is therefore desirable to avoid any a priori assumption of small perturbations from a Maxwellian. We are also particularly interested in the effect of perpendicular drifts, which may also result in strong anisotropy. In this approach one does not introduce $\overrightarrow{\boldsymbol{\Pi}} \equiv \ddot{\widetilde{\mathbf{P}}}-p \overrightarrow{\mathbf{I}}$, but instead directly expands $\overrightarrow{\mathbf{P}}$, which includes both average, $\mathbf{V}$, and random (thermal) velocities, $\tilde{\mathbf{v}}=\left(\tilde{\boldsymbol{v}}_{\perp}, \tilde{v}_{\|}\right)$, and which results in the appearance of two pressures, $p_{\perp}$ and $p_{\|}$in the momentum equation. The existence of two pressures $p_{\perp}$ and $p_{\|}$, is the most basic consequence of not assuming that 
collisionality is necessarily strong. Since $\vec{\Pi}$ does not appear in the momentum equation now, and since one can add the electron and ion momentum equations together, thus eliminating $R_{/ / e i}$, then in this approach the first two conservation equations are general as to the degree of collisionality. The closure problem is relegated to the energy equation(s); now two energy equations are required, one for $p_{\perp}$ and one for $p_{\|}$(for electrons and ions each). In this approach, and after adding the $e$ and $i$ momentum equations, collisionality makes its first explicit appearance in the energy equations in simple, transparently clear terms, e.g. $\left(p_{\perp}-\right.$ $\left.p_{\|}\right) / \tau_{i i}$, [44]'s Eq. (29), greatly facilitating the identification of appropriate equation sets for different degrees of collisionality: by taking $\tau_{i i} \rightarrow 0$, [44] recovered the principal terms in the strong collisionality equations of Braginskii, while taking $\tau_{i i} \rightarrow \infty$ they recovered the zero collisionality equations of Chew, Goldberger and Low, see Sec. 6. The [44] analysis does not include drifts or any other cross-field transport - therefore, when applied to the strong collisionality case, it recovers the collisional Braginskii transport coefficient estimate for $\Pi_{\|}$ (i.e. $\Pi_{z z}$ ) only; however, as shown by Braginskii, the other (collisional) components of $\vec{\Pi}$ are often smaller and so less important.

If is often preferred to avoid the additional computational effort involved with two energy equations, in which case $p$ and $\Pi_{\|}$can be used, rather than $p_{\perp}$ and $p_{\|}$; however, then some transport coefficient estimate is needed for $\Pi_{\|}$in the momentum equation, which can be derived in various ways, see $\mathrm{Sec} 6$. The standard approximations used to obtain an estimate, $\Pi_{\|}^{e s t}$, however, are only valid when collisionality is strong. The approach based on $\left(p, \Pi_{\|}^{e s t}\right)$ is taken, for example, in the major codes used for modelling the boundary region of magnetically-confined devices, e.g. EDGE2D [26], UEDGE [30], and B2.5 [35]. Weakly collisional plasmas are typical of the boundary region of present day, strongly heated tokamaks, however, and the values of $\Pi_{\|}^{\text {est }}$ often become unphysically large, requiring imposition of ad hoc 'kinetic limit factors', see Sec. 6. Continuing progress toward achievement of fusion power in devices such as the ITER tokamak motivates better approximations for the momentum equation, as can be achieved by using two pressures, i.e. allowing for the possibility of strong anisotropy of the ion distribution, the approach taken here.

We choose here to generally follow the approach of Zaweideh et al [44], since our principal interest is in drifts, which are a non-collisional effect, and so it is preferable to delay the introduction of collisionality assumptions into the analysis as long as possible. We differ from [44] in including the effect on the parallel momentum equation of non-parallel transport caused by drifts, anomalous cross-field transport and Larmor rotation. In common with [44] we will not assume that $\mathbf{B}$ is necessarily spatially constant, but in contrast with [44] we do assume $\mathbf{B}$ constant in time. Here, in order to bring out more explicitly and clearly the contribution of drifts, we will insert expressions for drift velocities given by Eq. (24) below directly into the total pressure-stress tensor $\overrightarrow{\mathbf{P}}=n m\langle\mathbf{v} \mathbf{v}\rangle$ in Section 4.

The direct approach to opening $\overrightarrow{\mathbf{P}}$ requires identification of the spatial variation of the components of the individual particle velocity vector, $\mathbf{v}=\left(v_{\perp_{1}}, v_{\perp_{2}}, v_{\|}\right)$, where ' $\|$' is the direction parallel to the magnetic field. The $v_{\perp_{1}}$-direction and $v_{\perp_{2}}$-directions are both perpendicular to $\mathbf{B}$ and to each other. Any pair of consistently defined perpendicular directions can be used and the results of the analysis here do not depend on any specific choice for them; however, here for the sake of concreteness (and familiarity) we will use ' $r$ ' 
in place of $v_{\perp_{1}}$ and ' $\mathrm{d}$ ' in place of $v_{\perp_{2}}$. In tokamak configurations, etc., ' $r$ ' is generally taken to be the 'radial' direction, i.e. perpendicular to the magnetic flux surface, while ' $d$ ' is the 'diamagnetic' direction, i.e. perpendicular to $\mathbf{B}$, but lying within the magnetic flux surface (less accurately this $3^{\text {rd }}$ direction is often called the 'poloidal' direction, which in tokamaks is often close to the diamagnetic direction due to smallness of the ratio of the poloidal to the total magnetic field). As noted, the results of the following analysis do not depend on any specific magnetic configuration, such as that of the tokamak, nor on the particular choice of the perpendicular coordinate pair $\left(\perp_{1}, \perp_{2}\right)$. The perpendicular velocity (by which we here understand the whole part of the velocity perpendicular to the magnetic field, i.e. $\left.\mathbf{v}_{\perp}=\left(v_{r}, v_{d}\right)\right)$, can also be split into four distinct parts:

$\mathbf{v}_{\perp}=\mathbf{v}_{\perp, L}+\mathbf{v}_{\perp, d r}+\mathbf{v}_{\perp, \text { anom }}+\mathbf{v}_{\perp, \text { coll }}$

In this paper, we denote individual particle velocities with small letters $\mathbf{v}$ and $v$. Here $\mathbf{v}_{\perp, L}$ describes fast Larmor rotation about the 'drifting' guiding centre of the Larmor orbit. As noted, we will drop this term. $\mathbf{v}_{\perp, d r}$ describes the perpendicular component of the guiding centre drift in a quasi-stationary electromagnetic field (see Eq. (24) below), $\mathbf{v}_{\perp \text {, anom }}$ is the perpendicular anomalous velocity due to e.g. the fluctuating electromagnetic field (which is usually characterized by much smaller frequencies than the frequency of the Larmor rotation) leading to anomalous turbulent transport, and $\mathbf{v}_{\perp \text {,coll }}$ gives the deviation from the Larmor rotation due to Coulomb collisions.

We defer to subsequent analysis inclusion of $\mathbf{v}_{\perp, \text { coll }}$, the most basic effects of which are covered by Braginskii's analysis; the resulting terms are small unless $\omega \tau$ is very small, where $\omega=e B / m$ and $\tau$ is the self-collisional time. Collisionality can vary significantly within the same plasma. For example, collisionality varies greatly in the edge of magnetic confinement devices, ranging from almost collisionless conditions that sometimes exist far from solid surfaces to extremely collisional conditions near tokamak divertor targets when plasma detachment [1] occurs and the role of $\mathbf{v}_{\perp, \text { coll }}$ can therefore be very important in tokamak edge modelling. In the extreme case of very cold, strongly collisional plasmas, the role of drifts in general is expected to be small, however, and the transport is dominated by parallel flow [5] and plasma-neutral interaction - therefore a natural separation of regimes often exists, to some degree.

The well known $\mathbf{v}_{\perp, d r}$-terms, i.e. the $\mathrm{E} \times \mathrm{B}, \mathrm{B} \times \nabla \mathrm{B}$ (or simply ' $\nabla \mathrm{B}$ ') and centrifugal guiding centre drifts, are key contributors to $\mathbf{v}_{\perp}$ (all contributions can be found in Eq. (24) below). Before inserting these $\mathbf{v}_{\perp, d r}$-terms into $\overrightarrow{\mathbf{P}}=n m\langle\mathbf{v v}\rangle$, which is done in Sec. 4, we must first carry out a coordinate transformation from rectangular coordinates to curvilinear $(r, d, \|)$ coordinates, in order to express $\overrightarrow{\mathbf{P}}=n m\langle\mathbf{v v}\rangle$ in a form appropriate for arbitrary magnetic geometry.

Our aim in this section is to provide a straightforward derivation in a convenient, physically clear form of the parallel component of the gradient of the total pressure-stress tensor. We take here a quite basic and general approach. The derivation involves operations with unit vectors of local (related to the direction of the local magnetic field) coordinate system, and it 
does not require use of tensor algebra, nor operations with metric coefficients. Such an approach doesn't require any specific assumptions about the magnetic geometry, e.g. any assumption about toroidal symmetry of the magnetic configuration. Although this overall undertaking was motivated by the specific application of the tokamak edge, the analysis here should be applicable to a wide range of conditions in magnetic confinement, space and astrophysical plasmas. The magnetic field is analyzed locally and is therefore independent of the overall magnetic structure and whether it is nested or not, toroidally symmetrical or not, reconnected or not, open or closed, etc. It is also precise in accounting for all convective and viscous terms involved (including averages of products of parallel and perpendicular velocities), since the derivation is based on the transformation of individual particle parallel and perpendicular velocity from one coordinate system to the other. Finally, the derivation is general as to the assumed degree of collisionality.

In a simple case of a Cartesian system of coordinates with unit direction vectors $\mathbf{i}_{x}, \mathbf{i}_{y}, \mathbf{i}_{z}$, and with the direction of the magnetic field $\mathbf{B}$ along $\mathbf{i}_{z}$, and field lines being straight and parallel to each other, the parallel component of the gradient of the tensor $(\vec{\nabla} \cdot \overrightarrow{\mathbf{P}})_{\|}$can be simplified by using the identity (note that $\mathbf{v v}$ is the dyadic product of 2 vectors, a dyad):

$$
(\vec{\nabla} \cdot \overrightarrow{\mathbf{P}})_{z} \equiv(\vec{\nabla} \cdot(n m\langle\mathbf{v} \mathbf{v}\rangle))_{z}=\vec{\nabla} \cdot\left(n m\left\langle v_{z} \mathbf{v}\right\rangle\right)
$$

We will also substitute the parallel direction ' $\|$ ' for ' $z$ '. Terms with parallel and perpendicular components of the velocity can then be easily separated. In the more general case of a curvilinear geometry, where parallel and perpendicular (with respect to the magnetic field) directions change in space, expressing the above quantity in terms of local parallel and perpendicular velocities is not so trivial. Eq. (3) still holds, but one cannot replace zsubscripts with the ' $\|$ ' sign.

We wish to open the tensor at some general point O, see Fig.1. Gradients such as $x(f)$ are involved, where $f$ is a quantity dependant on $x, y, z$ and ${ }_{x}(f)=$ $\left.\lim _{\Delta x \rightarrow 0} \frac{f(x=\Delta x)-f(x=0)}{\Delta x}\right|_{y, z \text { constant }}$, etc. Thus we need to evaluate $f$ at some point incrementally displaced from $\mathrm{O}$, call it $\Omega$, Fig. 1, i.e. $f(x=\Delta x)=f_{\Omega}$ and $\mathrm{F}_{x}(f)=\lim _{\Delta x \rightarrow 0} \frac{f_{\Omega}-f_{O}}{\Delta x}$. The coordinates of $\Omega$ are $(x, y, z)$. However, to emphasize that we need only consider incrementally small $x, y$ and $z$, we indicate them here as $(\Delta x, \Delta y, \Delta z) . \mathbf{v}$ is the velocity at $\mathrm{O}$ (thus also at $\Omega$, since we will take the limit $\Delta x \rightarrow 0$, etc.). We need to specify $\mathbf{v}$ in two different, orthogonal coordinate systems (see Fig. 1):

(a) local coordinate system, origin $O$ (but also $\Omega$ since we take the limit $\Delta x \rightarrow 0$, etc.), with unit direction vectors $\mathbf{i}_{r}, \mathbf{i}_{d}, \mathbf{i}_{\|}$where $\mathbf{i}_{\|}$is parallel to $\mathbf{B}, \mathbf{i}_{r}$ is perpendicular $\mathbf{B}$, and $\mathbf{i}_{d}$ is perpendicular to both $\mathbf{B}$ and $\mathbf{i}_{r}$. In this coordinate system the location of any general point is given by $\mathbf{r}=\left(\mathrm{s}_{r}, \mathrm{~s}_{d}, \mathrm{~s}_{\|}\right)=\mathrm{s}_{r} \mathbf{i}_{r}+\mathrm{s}_{d} \mathbf{i}_{d}+\mathrm{s}_{\|} \mathbf{i}_{\|}$and velocity $\mathbf{v}=\left(v_{r}, v_{d}, v_{\|}\right)$.

(b) a Cartesian, or rectangular, coordinate system with unit direction vectors $\mathbf{i}_{x}, \mathbf{i}_{y}, \mathbf{i}_{z}$. Here $\mathbf{r}=(x, y, z)$ and $\mathbf{v} \equiv\left(v_{x}, v_{y}, v_{z}\right)$. The origin of this coordinate system is point $\mathrm{O}$. At point $\mathrm{O}$, the Cartesian and local coordinate systems are identical 
We may also note that since increments are involved which $\rightarrow 0$, then $\mathrm{F}_{x}=\mathrm{F}_{r}, \mathrm{~F}_{y}=\mathrm{F}_{d}$ and $\mathrm{F}_{z}$ $=\mathrm{F}_{\|}$.

We define $\mathbf{b} \equiv \mathbf{B} / B$ and at point $\mathrm{O}, \mathbf{b}=\mathbf{b}_{\mathrm{o}}$ and $\mathbf{i}_{z} \| \mathbf{b}_{\mathrm{o}}$.

We want to express $(\vec{\nabla} \cdot \overrightarrow{\mathbf{P}})_{\|} \equiv(\vec{\nabla} \cdot(n m\langle\mathbf{v v}\rangle))_{\|}$ultimately in local velocity coordinates $\mathbf{v}=\left(v_{r}\right.$, $\left.v_{d}, v_{\|}\right)$, and we start by opening it in the Cartesian coordinate system using Eq. (3) (\| and $z$ directions, of course, coincide at the origin, point $\mathrm{O}$ ):

$$
\vec{\nabla} \cdot(n m\langle\mathbf{v v}\rangle))_{\|}=\partial_{z}\left(n m\left\langle v_{z}^{2}\right\rangle\right)+\partial_{x}\left(n m\left\langle v_{z} v_{x}\right\rangle\right)+\partial_{y}\left(n m\left\langle v_{z} v_{y}\right\rangle\right)
$$

We now relate $v_{x}, v_{y}, v_{z}$ to $v_{r}, v_{d}, v_{\|}$by specifying the transformation of coordinates, i.e. by relating $(x, y, z)$ to $\left(s_{r}, s_{d}, s_{\|}\right)$. While a state variable like $n$ is evaluated at $\left(s_{r}=0, s_{d}=0, s_{\|}=\right.$ 0 ), in order to evaluate the velocities $v_{r}, v_{d}, v_{\|}$, which are small spatial increments divided by a small time increment, we need to consider a location incrementally removed from $\Omega$, i.e. non-zero increments $s_{r}, s_{d}$, and $s_{\|}$. In order to express the velocity consider the trajectory in time of some particle whose location is $\mathbf{r}_{L}$. In the Cartesian coordinates $\mathbf{r}_{L}=\left(x_{L}, y_{L}, z_{L}\right)$ and in local coordinates $\mathbf{r}_{L}=\left(s_{r L}, s_{d L}, s_{\| L}\right)$, also $v_{x}=\frac{\partial x_{L}}{\partial t}$, etc. Then:

$$
\left\{\begin{array}{l}
x_{L}=\Delta x+\left(\mathbf{i}_{x} \cdot \mathbf{i}_{r}\right) s_{r L}+\left(\mathbf{i}_{x} \cdot \mathbf{i}_{d}\right) s_{d \mathrm{~L}}+\left(\mathbf{i}_{x} \cdot \mathbf{i}_{\|}\right) s_{\| L} \\
y_{L}=\Delta y+\left(\mathbf{i}_{y} \cdot \mathbf{i}_{r}\right) s_{r L}+\left(\mathbf{i}_{y} \cdot \mathbf{i}_{d}\right) s_{d \mathrm{~L}}+\left(\mathbf{i}_{y} \cdot \mathbf{i}_{\|}\right) s_{\| L} \\
z_{L}=\Delta z+\left(\mathbf{i}_{z} \cdot \mathbf{i}_{r}\right) s_{r L}+\left(\mathbf{i}_{z} \cdot \mathbf{i}_{d}\right) s_{d \mathrm{~L}}+\left(\mathbf{i}_{z} \cdot \mathbf{i}_{\|}\right) s_{\| L}
\end{array}\right.
$$

Time differentiation of Eq. (5) gives the relation between $\left(v_{x}, v_{y}, v_{z}\right)$ and $\left(v_{r}, v_{d}, v_{\|}\right)$, also involving, however, the (time-independent) directional cosines such as $\mathbf{i}_{x} \cdot \mathbf{i}_{r}$, which can themselves depend on $(\Delta x, \Delta y, \Delta z)$ and which we must now evaluate. Quantities $\Delta x, \Delta y$ and $\Delta z$ are not functions of time $t$.

Each unit vector of a local coordinate system, e.g. $\mathbf{i}_{r}$, can be expressed as a sum of its value at the origin ( $\mathbf{i}_{x}$, for the case of the vector $\mathbf{i}_{r}$ ) and an incremental change caused by the spatial displacement from $\mathrm{O}$ to $\Omega$. The latter can in turn be expressed through spatial derivatives:

$\mathbf{i}_{r}=\mathbf{i}_{x}+\Delta x \partial_{r} \mathbf{i}_{r}+\Delta y \partial_{d} \mathbf{i}_{r}+\Delta z \partial_{\|} \mathbf{i}_{r}$

In the limit $\mathrm{F}_{r} \mathbf{i}_{r}, \mathrm{~F}_{d} \mathbf{i}_{r}$ and $\mathrm{F}_{\|} \mathbf{i}_{r}$ in Eq. (6) coincide with $\mathrm{F}_{x} \mathbf{i}_{r}, \mathrm{~F}_{y} \mathbf{i}_{r}$ and $\mathrm{F}_{z} \mathbf{i}_{r}$.

An important feature of small angle rotations of the coordinate system, which we will be using extensively below, is that an incremental change in each unit vector, e.g. ( $\left.\mathbf{i}_{r}-\mathbf{i}_{x}\right)$, is almost perpendicular to the initial vector $\left(\mathbf{i}_{x}\right)$. The direction cosine $\left(\mathbf{i}_{x} \cdot \mathbf{i}_{r}\right)$ can be expressed through a (small) rotational angle $\alpha$ as $\cos \alpha \approx\left(1-\alpha^{2} / 2\right)$, hence $\cos \alpha \approx 1$ to first order. Thus in the limit $\left(\mathbf{i}_{x} \cdot \mathbf{i}_{r}\right)=1$, i.e. $\alpha$, and thus also $\Delta x, \Delta y$, and $\Delta z$, are only involved at $2^{\text {nd }}$ and higher orders. By multiplying Eq. (6) by $\mathbf{i}_{x}$ and taking into account (a) that $\left(\mathbf{i}_{x} \cdot \mathbf{i}_{x}\right)=1$ 
identically, (b) that the relation between $\mathbf{i}_{r}$ and $\mathbf{i}_{x}$, namely Eq. (6), involves $\Delta x, \Delta y$, and $\Delta \mathrm{z}$ to $1^{\text {st }}$ order and (c) that $\Delta x, \Delta y$, and $\Delta \mathrm{z}$ are independent variables, one then also obtains that:

$\mathbf{i}_{r} \cdot \partial_{r} \mathbf{i}_{r}=\mathbf{i}_{r} \cdot \partial_{d} \mathbf{i}_{r}=\mathbf{i}_{r} \cdot \partial_{\|} \mathbf{i}_{r}=0$

which we use below. Similar relations can be obtained for $\mathbf{i}_{d}$ and $\mathbf{i}_{\|}$unit vectors (although it should be noted that $i_{\|} \cdot \partial_{r} i_{r} \neq 0$, for example, because $\partial_{r} i_{r} \approx \sin \alpha \approx \alpha$ and so is first order in $\alpha$, and thus also in $\Delta x, \Delta y$, and $\Delta z$ ). In the Eq. (5) one can therefore assume for all 'diagonal' direction cosines:

$\left(\mathbf{i}_{x} \cdot \mathbf{i}_{r}\right),\left(\mathbf{i}_{y} \cdot \mathbf{i}_{d}\right),\left(\mathbf{i}_{z} \cdot \mathbf{i}_{\|}\right) \approx 1$

Thus $\quad \mathbf{i}_{\|} \equiv\left(b_{x}, b_{y}, b_{z}\right) \approx\left(b_{x}, \quad b_{y}, \quad 1\right), \quad \mathbf{i}_{r} \quad \equiv\left(\mathbf{i}_{r} \cdot \mathbf{i}_{x}, \mathbf{i}_{r} \cdot \mathbf{i}_{y}, \mathbf{i}_{r} \cdot \mathbf{i}_{z}\right) \approx(1, \quad \mathrm{C}, \quad \mathrm{D}), \quad \mathbf{i}_{d}$ $\equiv\left(\mathbf{i}_{d} \cdot \mathbf{i}_{x}, \mathbf{i}_{d} \cdot \mathbf{i}_{y}, \mathbf{i}_{d} \cdot \mathbf{i}_{z}\right) \approx(\mathrm{E}, 1, \mathrm{~F})$ where the unit vector $\mathbf{b}=\mathbf{B} / B$. The quantities $\mathrm{C}, \mathrm{D}, \mathrm{E}, \mathrm{F}$ are of order $b_{x}, b_{y}$ and can be found by using $\mathbf{i}_{r}=\mathbf{i}_{d} \times \mathbf{i}_{\|}$and $\mathbf{i}_{d}=\mathbf{i}_{\|} \times \mathbf{i}_{r}$. One can therefore obtain: $\mathbf{i}_{r}$ $\approx\left(1, \mathrm{C},-b_{x}\right), \mathbf{i}_{d} \approx\left(-\mathrm{C}, 1,-b_{y}\right)$, where $\mathrm{C}$ cannot be simply expressed in terms of $b_{x}$ and $b_{y}$ (which turns out not to be needed anyway, below), resulting in:

$\left(\mathbf{i}_{x} \cdot \mathbf{i}_{\|}\right) \approx b_{x},\left(\mathbf{i}_{y} \cdot \mathbf{i}_{\|}\right) \approx b_{y}$,

$\left(\mathbf{i}_{z} \cdot \mathbf{i}_{r}\right)=-\left(\mathbf{i}_{\|} \cdot \mathbf{i}_{x}\right) \approx-b_{x}$

$\left(\mathbf{i}_{z} \cdot \mathbf{i}_{d}\right)=-\left(\mathbf{i}_{\|} \cdot \mathbf{i}_{y}\right) \approx-b_{y}$

As just noted, the remaining two direction cosines: $\left(\mathbf{i}_{y} \cdot \mathbf{i}_{r}\right)$ and $\left(\mathbf{i}_{x} \cdot \mathbf{i}_{d}\right)$, have equal absolute values but opposite signs. Using the relations for the direction cosines obtained earlier the transformation of velocity components which follows directly from Eq. (5) can be cast into the form:

$\left\{\begin{array}{l}v_{x} \approx v_{r}+\left(\mathbf{i}_{x} \cdot \mathbf{i}_{d}\right) v_{d}+b_{x} v_{\|} \\ v_{y} \approx\left(\mathbf{i}_{y} \cdot \mathbf{i}_{r}\right) v_{r}+v_{d}+b_{y} v_{\|} \\ v_{z} \approx-b_{x} v_{r}-b_{y} v_{d}+v_{\|}\end{array}\right.$

Using Eq. (6) one can express $\mathbf{i}_{x}$ through $\mathbf{i}_{r}$. Taking the dot product of $\mathbf{i}_{d}$ and $\mathbf{i}_{r}$ from Eq. (6) and taking into account that $\left(\mathbf{i}_{r} \cdot \mathbf{i}_{d}\right)=0$ one obtains:

$\left(\mathbf{i}_{x} \cdot \mathbf{i}_{d}\right)=-\Delta x \mathbf{i}_{d} \cdot \partial_{r} \mathbf{i}_{r}-\Delta y \mathbf{i}_{d} \cdot \partial_{d} \mathbf{i}_{r}-\Delta z \mathbf{i}_{d} \cdot \partial_{\|} \mathbf{i}_{r}$

Similarly, for the other direction cosine in Eq. (10) one can obtain:

$\left(\mathbf{i}_{y} \cdot \mathbf{i}_{r}\right)=-\Delta x \mathbf{i}_{r} \cdot \partial_{r} \mathbf{i}_{d}-\Delta y \mathbf{i}_{r} \cdot \partial_{d} \mathbf{i}_{d}-\Delta z \mathbf{i}_{r} \cdot \partial_{\|} \mathbf{i}_{d}$

We now substitute (10), (11) and (12) into (4), neglecting in the limit all terms containing $b_{x}$, $b_{y}, \Delta x, \Delta y$, and $\Delta z$, but retaining their gradients, and taking into account that $\mathrm{F}_{x} \Delta x=\mathrm{F}_{y} \Delta y=1$ and $\mathrm{F}_{x} \Delta y=\mathrm{F}_{x} \Delta z=\mathrm{F}_{y} \Delta x=\mathrm{F}_{y} \Delta z=0$ to obtain: 


$$
\begin{aligned}
(\vec{\nabla} \cdot \overrightarrow{\mathbf{P}})_{\|} & =\partial_{\|}\left(n m\left\langle v_{\|}^{2}\right\rangle\right)-2 n m\left\langle v_{\|} v_{r}\right\rangle\left(\partial_{z} b_{x}\right)-2 n m\left\langle v_{\|} v_{d}\right\rangle\left(\partial_{z} b_{y}\right)+\partial_{x}\left(n m\left\langle v_{\|} v_{r}\right\rangle\right) \\
& -n m\left\langle v_{r} v_{d}\right\rangle\left(\partial_{y} b_{x}\right)-n m\left\langle v_{r} v_{\mathrm{d}}\right\rangle\left(\partial_{x} b_{y}\right)+\partial_{y}\left(n m\left\langle v_{\|} v_{d}\right\rangle\right)+n m\left\langle v_{\|}^{2}-\mathrm{v}_{d}^{2}\right\rangle\left(\partial_{y} b_{y}\right) \\
+ & n m\left\langle v_{\|}^{2}-\mathrm{v}_{r}^{2}\right\rangle\left(\partial_{x} b_{x}\right)-n m\left\langle v_{\|} v_{d}\right\rangle\left(\mathbf{i}_{d} \cdot \partial_{r} \mathbf{i}_{r}\right)-n m\left\langle v_{\|} v_{r}\right\rangle\left(\mathbf{i}_{r} \cdot \partial_{d} \mathbf{i}_{d}\right)
\end{aligned}
$$

In the above equation all $x, y$ and $z$-derivatives can be replaced with corresponding $r, d$ and $\|$ - derivatives, as already noted. Such a replacement will make this equation be fully written in local coordinates, except for the components of the unit vector $\mathbf{b}$ which must, of course, be defined in the Cartesian system of coordinates. In the above equation $\mathrm{F}_{z} b_{x}$ and $\mathrm{F}_{z} b_{y}$ can be replaced with $\mathrm{F}_{\|} b_{x}$ and $\mathrm{F}_{\|} b_{y}$, respectively.

Up to this point in the analysis, $v_{r}$ and $v_{d}$ are general and include all components of the perpendicular velocity $\mathrm{v}_{\perp}=\mathrm{v}_{\perp, d r}+\mathrm{v}_{\perp \text {, anom }}+\mathrm{v}_{\perp \text {, coll }}$ where $\mathbf{v}_{\perp}=\left(v_{r}, v_{d}\right)$. With little significant loss of generality, however, we may take it that all the terms with velocities squared on the RHS of equation (13) are large compared to other terms, since their averaging over the distribution function gives pressures, to a good approximation. Averages $\left\langle v_{r}^{2}\right\rangle$ and $\left\langle v_{d}^{2}\right\rangle$ can be represented by sums of the corresponding random and mean parts, e.g.: $\left\langle v_{\perp}^{2}\right\rangle=\left(\left\langle\tilde{v}_{\perp}^{2}\right\rangle+V_{\perp}^{2}\right)$ and we may, with little loss of generality, assume that $\left\langle\tilde{v}_{\perp}^{2}\right\rangle \approx\left\langle\tilde{v}_{r}^{2}\right\rangle \approx v_{t h}^{2}$, since thermal speeds, $v_{t h}$, greatly exceed all other $v_{\perp}$ speeds, typically. The first part accounts for chaotic (thermal) energy, and it coincides with $\left\langle\tilde{v}_{r}^{2}\right\rangle$. The second part is much smaller, involving $\mathrm{E} \times \mathrm{B}, \nabla \mathrm{B}$, etc. drifts. We will be neglecting terms $V_{r}^{2}, V_{d}^{2}$ compared with some other terms that we will retain. The terms $\left\langle v_{r}^{2}\right\rangle$ and $\left\langle v_{d}^{2}\right\rangle$ can therefore be considered equal to each other and both can be expressed as $\left\langle v_{\perp}^{2}\right\rangle / 2$. Other terms that can be neglected are the ones with $\left\langle v_{r} v_{d}\right\rangle$. In this average, the main, chaotic parts are uncorrelated (particles have random distributions of their Larmor rotation phases), so it can be roughly estimated as $V_{r} V_{d}$, with both velocities in this product being much smaller than thermal velocities, as shown above.

After neglecting small terms, replacing $\left\langle v_{r}^{2}\right\rangle$ and $\left\langle v_{d}^{2}\right\rangle$ with $\left\langle v_{\perp}^{2}\right\rangle / 2$, re-arranging positions of individual terms and replacing $x, y$ and $z$-derivatives with $r, d$ and $\|$ - derivatives, where appropriate, Eq. (13) becomes:

$$
\begin{array}{r}
(\vec{\nabla} \cdot \overrightarrow{\mathrm{P}})_{\|}=\partial_{\|}\left(n m\left\langle v_{\|}^{2}\right\rangle\right)+n m\left\langle v_{\|}^{2}-v_{\perp}^{2} / 2\right\rangle\left(\partial_{x} b_{x}+\partial_{y} b_{y}\right)+\partial_{r}\left(n m\left\langle v_{\|} v_{r}\right\rangle\right)+\partial_{d}\left(n m\left\langle v_{\|} v_{d}\right\rangle\right) \\
-2 n m\left\langle v_{\|} v_{r}\right\rangle\left(\partial_{\|} b_{x}\right)-2 n m\left\langle v_{\|} v_{d}\right\rangle\left(\partial_{\|} b_{y}\right)-n m\left\langle v_{\|} v_{d}\right\rangle\left(\mathrm{i}_{d} \cdot \partial_{r} \mathrm{i}_{r}\right)-n m\left\langle v_{\|} v_{r}\right\rangle\left(\mathrm{i}_{r} \cdot \partial_{d} \mathrm{i}_{d}\right)
\end{array}
$$

We wish to re-write this equation further to bring out the significance of the various terms and to make the equation more compact. We start with the $2^{\text {nd }}$ term on the RHS of Eq. (14) and the quantity $\left(\partial_{x} b_{x}+\partial_{y} b_{y}\right)$. Since $b_{z}$ can be expressed as $\sqrt{1-b_{x}^{2}-b_{y}^{2}} \approx\left(1-b_{x}^{2} / 2-b_{y}^{2} / 2\right) \approx 1$, then $\partial_{z} b_{z}=0$ and $\left(\partial_{x} b_{x}+\partial_{y} b_{y}\right)$ gives the full 
divergence of the unit vector $\mathbf{b}$, $\operatorname{div} \mathbf{b}$. Using $\operatorname{div} \mathbf{B}=\operatorname{div}(B \mathbf{b})=\vec{\nabla} B \cdot \mathbf{b}+B \operatorname{div} \mathbf{b}=0$, one obtains: $\operatorname{div} \mathbf{b}=-\mathbf{b} \cdot \vec{\nabla} B / B=-\partial_{\|} B / B$.

Next we consider the $5^{\text {th }}$ and $6^{\text {th }}$ terms on the RHS of Eq. (14) and the quantities $\partial_{\|} b_{x}$ and $\partial_{\|} b_{y}$. From elementary theory for vector functions and 3D space curves, one of the FermatSerret formulas gives $d \mathbf{T} / d s_{\|}=k \mathbf{N}$ where $\mathbf{T}$ is the unit tangent vector, $k$ is the curvature $(k=$ $1 / R_{c}$ where $R_{c}$ is the radius of curvature) and $\mathbf{N}$ is the unit normal vector, $\mathbf{N}=-\mathbf{R}_{c} / R_{c}$ where $-\mathbf{R}_{c}$ points toward the centre of curvature. The curvature vector $\mathbf{k}=k \mathbf{N}=-\mathbf{R}_{c} / R_{c}^{2}$. Here $\mathbf{T}=\mathbf{b}$ thus $\partial_{\|} \mathbf{b}=\mathbf{k}$. Therefore $\partial_{\|} b_{x}$ can be expressed as $\partial_{\|}\left(\mathbf{b} \cdot \mathbf{i}_{x}\right)=\mathbf{i}_{x} \cdot \partial_{\|} \mathbf{b}$ (since $\mathbf{i}_{x}$ is a unit vector constant in space) and further as $\mathbf{i}_{x} \cdot \mathbf{k}=k_{x}$, which is the same as $k_{r}$ at the origin of the Cartesian coordinate system. Similarly, $\partial_{\|} b_{y}$ can be replaced with $k_{y}=k_{d}$.

We will now group a sub-set of the terms in Eq. (14) into a quantity we will call $-F_{d i v}$, which we will then show is simply $\vec{\nabla} \cdot\left(n m\left\langle v_{\|} \mathbf{v}_{\perp}\right\rangle\right)$. The term $\partial_{r}\left(n m\left\langle v_{\|} v_{r}\right\rangle\right)$ can be combined with half of the $5^{\text {th }}$ term on the RHS of Eq. (14), $-n m\left\langle v_{\|} v_{r}\right\rangle k_{r}$, to give $\left[\partial_{r}\left(n m\left\langle v_{\|} v_{r}\right\rangle\right)-n m\left\langle v_{\|} v_{r}\right\rangle k_{r}\right]$. Handling $y$ terms in the same way, one can obtain a similar combination, $\left[\partial_{d}\left(n m\left\langle v_{\|} v_{d}\right\rangle\right)-n m\left\langle v_{\|} v_{d}\right\rangle k_{d}\right]$. The sum of these two combinations, plus the last two terms in the Eq. (14), is:

$$
\begin{aligned}
-F_{d i v}= & \partial_{r}\left(n m\left\langle v_{\|} v_{r}\right\rangle\right)-n m\left\langle v_{\|} v_{r}\right\rangle k_{r}+\partial_{d}\left(n m\left\langle v_{\|} v_{d}\right\rangle\right)-n m\left\langle v_{\|} v_{d}\right\rangle k_{d} \\
& -n m\left\langle v_{\|} v_{d}\right\rangle\left(\mathbf{i}_{d} \cdot \partial_{r} \mathbf{i}_{r}\right)-n m\left\langle v_{\|} v_{r}\right\rangle\left(\mathbf{i}_{r} \cdot \partial_{d} \mathbf{i}_{d}\right)
\end{aligned}
$$

We note, without derivation, that a similar analysis of the particle equation gives for the divergence term there:

$$
\begin{aligned}
\vec{\nabla} \cdot(n \mathbf{V})=\partial_{r}\left(n V_{r}\right)+\partial_{d}\left(n V_{d}\right) & +\partial_{\|}\left(n V_{\|}\right)-n \mathbf{V}_{\perp} \cdot \mathbf{k}-n V_{\|}\left(\nabla_{\|} B / B\right) \\
& -n V_{d}\left(\mathbf{i}_{d} \cdot \partial_{r} \mathbf{i}_{r}\right)-n V_{r}\left(\mathbf{i}_{r} \cdot \partial_{d} \mathbf{i}_{d}\right)
\end{aligned}
$$

It can be shown that the above expression for $F_{d i v}$ represents the full divergence of the $n m\left\langle v_{\|} \mathbf{v}_{\perp}\right\rangle$ momentum flux: $-F_{d i v}=\vec{\nabla} \cdot\left(n m\left\langle v_{\|} \mathbf{v}_{\perp}\right\rangle\right)$. Indeed, by writing the full gradient operator as $\vec{\nabla}=\left(\mathbf{i}_{\|} \partial_{\|}+\mathbf{i}_{r} \partial_{r}+\mathbf{i}_{d} \partial_{d}\right)$, the full perpendicular velocity as $\mathbf{v}_{\perp}=\left(\mathbf{i}_{r} v_{r}+\mathbf{i}_{d} v_{d}\right)$ and performing scalar multiplications of individual vector terms, $\vec{\nabla} \cdot\left(n m\left\langle v_{\|} \mathbf{v}_{\perp}\right\rangle\right)$ can be expanded into the sum of the following six terms:

$$
\begin{aligned}
& \mathbf{i}_{\|} \cdot \partial_{\|}\left(\mathbf{i}_{r} n m\left\langle v_{\|} v_{r}\right\rangle\right), \\
& \mathbf{i}_{\|} \cdot \partial_{\|}\left(\mathbf{i}_{d} n m\left\langle v_{\|} v_{d}\right\rangle\right), \\
& \mathbf{i}_{r} \cdot \partial_{r}\left(\mathbf{i}_{r} n m\left\langle v_{\|} v_{r}\right\rangle\right), \\
& \mathbf{i}_{d} \cdot \partial_{d}\left(\mathbf{i}_{d} n m\left\langle v_{\|} v_{d}\right\rangle\right), \\
& \mathbf{i}_{r} \cdot \partial_{r}\left(\mathbf{i}_{d} n m\left\langle v_{\|} v_{d}\right\rangle\right),
\end{aligned}
$$


$\mathbf{i}_{d} \cdot \partial_{d}\left(\mathbf{i}_{r} n m\left\langle v_{\|} v_{r}\right\rangle\right)$

The first term here can be further expanded into the sum:

$\left[\mathbf{i}_{\|} \cdot \mathbf{i}_{r} \partial_{\|}\left(n m\left\langle v_{\|} v_{r}\right\rangle\right)+\mathbf{i}_{\|} \cdot n m\left\langle v_{\|} v_{r}\right\rangle \partial_{\|} \mathbf{i}_{r}\right]$

where the first part is equal to zero, while the second part can be transformed by using $\mathbf{i}_{r} \cdot \mathbf{i}_{\|}=0$, thus $\mathbf{i}_{\|} \cdot \partial_{\|} \mathbf{i}_{r}=-\mathbf{i}_{r} \cdot \partial_{\|} \mathbf{i}_{\|}$and remembering that $\mathbf{i}_{\|} \equiv \mathbf{b}$ (in our local coordinate system) and $\partial_{\|} \mathbf{b}=\mathbf{k}$ into $-n m\left\langle v_{\|} v_{r}\right\rangle k_{r}$, which is the second term on the RHS of Eq. (15). Similarly, the second term, $\mathbf{i}_{\|} \cdot \partial_{\|}\left(\mathbf{i}_{d} n m\left\langle v_{\|} v_{d}\right\rangle\right)$, will contribute the fourth term on the RHS in this equation. The third term, $\mathbf{i}_{r} \cdot \partial_{r}\left(\mathbf{i}_{r} n m\left\langle v_{\|} v_{r}\right\rangle\right)$, can be expanded into:

$\left[\partial_{r}\left(n m\left\langle v_{\|} v_{r}\right\rangle\right)+\mathbf{i}_{r} \cdot n m\left\langle v_{\|} v_{r}\right\rangle \partial_{r} \mathbf{i}_{r}\right]$

While the first part of this expression coincides with the first term on the RHS of Eq. (15), the second part is zero since $\mathbf{i}_{r} \cdot \partial_{r} \mathbf{i}_{r}$ is zero (Eq. (7)).

Similarly, the fourth term, $\mathbf{i}_{d} \cdot \partial_{d}\left(\mathbf{i}_{d} n m\left\langle v_{/ /} v_{d}\right\rangle\right)$, will only contribute the third term on the RHS of Eq. (15). The fifth term, $\mathbf{i}_{r} \cdot \partial_{r}\left(\mathbf{i}_{d} n m\left\langle v_{\|} v_{d}\right\rangle\right)$, can be expanded into the sum:

$\left[\mathbf{i}_{r} \cdot \mathbf{i}_{d} \partial_{r}\left(\left(n m\left\langle v_{\|} v_{r}\right\rangle\right)+\mathbf{i}_{r} \cdot\left(n m\left\langle v_{\|} v_{d}\right\rangle\right) \partial_{r} \mathbf{i}_{d}\right]\right.$

where the first part is zero while the second part, after taking into account that $\mathbf{i}_{r} \cdot \partial_{r} \mathbf{i}_{d}=-\mathbf{i}_{d} \cdot \partial_{r} \mathbf{i}_{r}$, becomes identical to the fifth term on the RHS of Eq. (15). Similarly, the sixth term, $\mathbf{i}_{d} \cdot \partial_{d}\left(\mathbf{i}_{r} n m\left\langle v_{\|} v_{r}\right\rangle\right)$, after the expansion into:

$\left[\mathbf{i}_{d} \cdot \mathbf{i}_{r} \partial_{d}\left(\left(n m\left\langle v_{\|} v_{r}\right\rangle\right)+\mathbf{i}_{d} \cdot\left(n m\left\langle v_{\|} v_{r}\right\rangle\right) \partial_{d} \mathbf{i}_{r}\right]\right.$

and using $\mathbf{i}_{d} \cdot \partial_{d} \mathbf{i}_{r}=-\mathbf{i}_{r} \cdot \partial_{d} \mathbf{i}_{d}$, coincides with the last term in Eq. (15).

The remaining terms (halves of the fifth and sixth terms in Eq. (14)) can also be written as $-\left(n m\left\langle v_{\|} v_{r}\right\rangle k_{r}+n m\left\langle v_{\|} v_{d}\right\rangle k_{d}\right)$ and, in the vector form, as:

$-F_{\text {curv }}=-n m\left\langle v_{\|} \mathbf{v}_{\perp}\right\rangle \cdot \mathbf{k}$

Finally, by replacing $n m\left\langle v_{\perp}^{2}\right\rangle / 2$ with $p_{\perp}$ and splitting $n m\left\langle v_{\|}^{2}\right\rangle$ into parts $n m V_{\|}^{2}$ and $p_{\|}$, Eq. (14) can be cast into the following form:

$(\vec{\nabla} \cdot \overrightarrow{\mathbf{P}})_{\|}=\nabla_{\|}\left(n m V_{\|}^{2}+p_{\|}\right)-\left(n m V_{\|}^{2}+p_{\|}-p_{\perp}\right) \nabla_{\|} B / B-F_{\text {div }}-F_{\text {curv }}$

where $F_{d i v}=-\vec{\nabla} \cdot\left(n m\left\langle v_{\|} \mathbf{v}_{\perp}\right\rangle\right), F_{c u r v}=n m\left\langle v_{\|} \mathbf{v}_{\perp}\right\rangle \cdot \mathbf{k}$ and $\nabla_{\|} \equiv \partial_{\|}$. 
It may be noted that $v_{r}$ and $v_{d}$ do not appear in Eq. (23) and therefore this result is general and independent of the specific choice of perpendicular coordinate pair $\left(\perp_{1}, \perp_{2}\right)$. Eq. (23) is also general as to the level of collisionality.

One may note that since the total pressure-stress tensor is involved here, there are both convective components as well as viscous ones in $(\vec{\nabla} \cdot \overrightarrow{\mathbf{P}})_{\|}$. Some of the convective components are already explicit, i.e. the $V_{\|}$terms - while others are implicit in the last two terms since $v_{\|} \equiv V_{\|}+\tilde{v}_{\|}$and $\mathbf{v}_{\perp} \equiv \mathbf{V}_{\perp}+\tilde{\mathbf{v}}_{\perp}$.

Eq. (23) does not contain the traditional viscous term proportional to the second derivative of the parallel velocity with respect to the parallel coordinate. Therefore well-developed methods for the integration of equations of the second order are not applicable here.

We now discuss the physical meaning of different terms in Eq. (23). The first term, $\nabla_{\|}\left(n m V_{\|}^{2}+p_{\|}\right)$, is obvious and follows from a $1 \mathrm{D}$ equilibrium along the field lines in a simple geometry, and with neglect of perpendicular gradients of the plasma parameters. The second term, $-\left(n m V_{\|}^{2}+p_{\|}-p_{\perp}\right) \nabla_{\|} B / B$, can be found e.g. in Refs. [44] (Eq. (17)) and [45] (Eqs. (1.27) and (1.28)). The physical interpretation of this term has been given in Ref. [45] by considering the force balance of an ensemble of Larmor circles along a flux tube with variable cross-section. The part $-\left(n m V_{\|}^{2}+p_{\|}\right) \nabla_{\|} B / B$ comes from the difference in crosssections $S$ of a flux tube at its ends, which is inversely proportional to the magnetic field: $S \sim 1 / B$ (hence, the appearance of $\nabla_{\|} B / B$ ), whereas $p_{\perp} \nabla_{\|} B / B$ comes from the projection of the volume force $-\mu \vec{\nabla} B$ exerted on each Larmor circle inside the tube ( $\mu=m v_{\perp}^{2} / 2 B$ is the magnetic moment of a Larmor circle) on the parallel direction.

The next term, $\vec{\nabla} \cdot\left(n m\left\langle v_{\|} \mathbf{v}_{\perp}\right\rangle\right)$, accounts for perpendicular flux of parallel momentum across the flux tube's lateral sides. Any actual computation, of course, requires an expansion of this compact expression and this is given by Eq. (15). It includes both the terms $\frac{\partial}{\partial s_{r}}\left(n m\left\langle v_{\|} v_{r}\right\rangle\right)$ and $\frac{\partial}{\partial s_{d}}\left(n m\left\langle v_{\|} v_{d}\right\rangle\right)$, which are intuitively clear contributions and the only ones present in the rectangular coordinate system, as well as four other terms appearing due to operations with the unit vectors of an arbitrary curvilinear coordinate system. Two of the latter involve curvature $\mathbf{k}$ but, being due to divergence of the flow field, have a different origin than the last term $F_{\text {curv }}=n m\left\langle v_{\|} \mathbf{v}_{\perp}\right\rangle \cdot \mathbf{k}$, considered next.

The parallel force $n m\left\langle v_{\|} \mathbf{v}_{\perp}\right\rangle \cdot \mathbf{k}$ is a volume force exerted on each particle within the flux tube. Similar to the $\mu \nabla B$ force acting on a Larmor circle (where parallel energy is transformed into perpendicular energy and vice versa, leading to conservation of the magnetic moment $\mu$ ) whose parallel projection accelerates the Larmor circle along the field lines, it accounts for mutual transformation between parallel and perpendicular energies in the curvilinear magnetic geometry. However, whereas this transformation in the case of the $\mu \nabla \mathrm{B}$ force is related to convergence (or divergence) of the field lines and is expressed via $\operatorname{div} \mathbf{b}$, the $n m\left\langle v_{\|} \mathbf{v}_{\perp}\right\rangle \cdot \mathbf{k}$ force originates due to the bending of the field lines, described by 
their curvature k. From simple geometrical considerations, it is readily shown that the rate of change of parallel momentum due to curvature $k=1 / R_{c}$ is $-m v_{\|} v_{\perp} / R_{c}$.

\section{Drift fluxes calculated from individual particle analysis}

In this section we will obtain expressions for $\mathbf{v}_{\perp, d r}$ from single particle analysis for insertion into $\overrightarrow{\mathbf{P}}=n m\langle\mathbf{v v}\rangle$, opened in the previous section.

In the drift approximation particle orbits are described as a superposition of Larmor rotation and the motion of the Larmor circle's centre ('guiding centre'). The guiding centre velocity in a quasi-stationary electromagnetic field (which we will refer to as 'drift velocity') is calculated from analysis of individual particle motion, smoothed or averaged over the Larmor rotation, and is given by (see e.g. Eq. (5.4) of [40], also Eq. (6.1) of [46]):

$$
\mathbf{v}_{d r}=\mathbf{b}\left(\bar{v}_{\|}+\frac{1}{2} \frac{m \bar{v}_{\perp}^{2}}{Z e B} \mathbf{b} \cdot c u r l \mathbf{b}\right)+\frac{\mathbf{E} \times \mathbf{B}}{B^{2}}+\frac{1}{2} \frac{m \bar{v}_{\perp}^{2}}{Z e B^{3}} \mathbf{B} \times \nabla B+\frac{m \bar{v}_{\|}^{2}}{Z e B^{3}} \mathbf{B} \times(\mathbf{B} \cdot \nabla \mathbf{b})
$$

where $\mathbf{b}=\mathbf{B} / B$ is the unit vector along the magnetic field and other notations are standard. Often the notation $\mathbf{v}_{\text {g.c. }}$ is used for this guiding centre (g.c.) drift velocity. We will, however, reserve $\mathbf{v}_{\text {g.c. }}$ to mean the time-averaged guiding centre velocity which also includes anomalous transport (which in turn includes drift velocity due to fluctuating electric fields), while $\mathbf{v}_{d r}$ will be understood here to be the guiding centre drift velocity in the quasistationary electromagnetic field (see Eq. (2)). In Eq. (24), $\bar{v}_{\|}$and $\bar{v}_{\perp}$ are not instant particle parallel and perpendicular velocities, but, as noted, their values averaged over the period of the Larmor rotation (with $\bar{v}_{\perp}$ being essentially the Larmor rotation velocity, $\bar{v}_{\perp, L}$, see below).

Components of the drift velocity are projected onto the magnetic field direction at the Larmor guiding centre, rather than at its direction at the particle's actual location. This explains the appearance of the term with (b-curlb) in Eq. (24). In most practical cases this term can be neglected for edge plasmas compared with $v_{\|}$(unless supra-thermal highly accelerated particles are considered). The ratio of the two terms is of order $\frac{\bar{v}_{\perp}}{\bar{v}_{\|}} \frac{\rho_{i}}{L_{B}}$, a very small number, where $\rho_{i}$ is the Larmor radius and $L_{B}$ is the scale size of spatial variation of the magnetic field structure. In the more approximate Eq. (5.5) of [46], for example, this term is neglected. We will, however, retain this term. We note that it is a parallel drift. The second, third and fourth terms on the RHS of Eq. (24) are the well known perpendicular drifts - the ExB, $\underline{\mathrm{B}} \times \nabla \mathrm{B}$ (or simply $\nabla \mathrm{B}$ ) and centrifugal drifts - respectively. In Eq. (24) $\bar{v}_{\perp}^{2}$ can, to within a good approximation (of order of a square of the ratio of drift velocity to thermal velocity, as can be easily demonstrated), be replaced with $\bar{v}_{\perp, L}^{2}$, owing to smallness of the drift velocity compared to the Larmor velocity, which is of the order of thermal velocity $v_{t h}$. 
From Eq. (24) we can anticipate two different types of perpendicular drift contribution to the total pressure-stress tensor $\overrightarrow{\mathbf{P}}=n m\langle\mathbf{v v}\rangle$, namely convective ones, which involve products of $V_{\|}$and simple averages of the velocities of the 3 perpendicular drifts - and viscous ones. To calculate the latter it is necessary to take into account that each individual particle has an individual value of (Larmor-orbit-averaged) parallel speed $\bar{v}_{\|}$and an individual (Larmororbit-averaged) perpendicular drift speed $\bar{v}_{\perp}$, and to then construct averages of the product of the two speeds over the distribution of the speeds. From Eq. (24) it is clear that ExB drifts will not contribute to the viscous part of $\overrightarrow{\mathbf{P}}$ but the other 2 drifts will, since each individual ion has an individual value of $\bar{v}_{\|}^{2}$ and $\bar{v}_{\perp}^{2}$, thus an individual value for each of these drift speeds. While $\bar{v}_{\perp} \approx v_{\perp, L}$ can thus appear in the viscous part of $\overrightarrow{\mathbf{P}}$ it is not because of any direct presence of Larmor rotation velocity in $\langle\mathbf{v v}\rangle$, as will be demonstrated in [42], but only because $\bar{v}_{\perp}$ appears within the expression for one of the perpendicular drift speeds, namely $\frac{1}{2} \frac{m \bar{v}_{\perp}^{2}}{Z e B^{3}} \mathbf{B} \times \nabla B$, and the latter does appear directly in $\langle\mathbf{v v}\rangle$. Therefore for straight, constant B, $\bar{v}_{\perp, L}$ will not appear at all in the expression for $\overrightarrow{\mathbf{P}}$, even indirectly.

In the standard neoclassical theory, developed for the core region (see e.g. $[43,47]$ ), the three main (perpendicular) drift velocities figuring in Eq. (24) are all roughly of the same order, assuming equal ion and electron temperatures, radial electric field $E_{r}$ to be of order $T_{i} / e r$, and considering minor radius $r$ and major radius $R$ to be of the same order. In the $\delta$-expansion (expansion in the ratio of the Larmor radius to either the scale size of spatial variation of the plasma parameters, or the $L_{B}$ size mentioned above), parallel velocities appear to be first order in $\delta$ (unless large toroidal momentum is introduced into the plasma by e.g. neutral beam injection, which is a separate issue not covered by the standard theory). At the plasma edge, and in particular in the SOL, the situation is quite different in several respects. For example, individual drifts of Eq. (24) are quite different from each other as to their magnitude (e.g. $\nabla \mathrm{B}$ drift velocity is much smaller then the ExB drift velocity). In Appendix A simple estimates are made for typical values of the drifts in the SOL of magnetically-confined devices such as tokamaks.

To summarize the findings from this section and Appendix A, we conclude that some technical procedures and conclusions of the standard neoclassical theory may prove to be incorrect for the plasma edge region. In the remainder of the paper, we will carry out the analysis by using terms with the best possible practical accuracy, comparing them with each other on a one-by-one basis, before making simplifications and drawing conclusions. This is particularly important in simplifying the expression for the gradient of the total pressurestress tensor that enters the parallel momentum balance equations, considered in the next section.

\section{Formulation of particle and parallel momentum fluid equations}

Eq. (23) gives the expression for the opened total pressure-stress tensor, where $p_{\|}=n m\left(\left\langle v_{\|}^{2}\right\rangle-V_{\|}^{2}\right)$ and $p_{\perp}=n m\left\langle v_{\perp}^{2}\right\rangle / 2$ are parallel and perpendicular pressures, respectively, $\nabla_{\|}=\partial / \partial \mathrm{s}_{\|}$is the scalar gradient along the parallel direction, 
$F_{\text {div }}=-\vec{\nabla} \cdot\left(n m\left\langle v_{\|} \mathbf{v}_{\perp}\right\rangle\right), F_{\text {curv }}=n m\left\langle v_{\|} \mathbf{v}_{\perp}\right\rangle \cdot \mathbf{k}$, and $\mathbf{k}$ is the curvature of the magnetic field lines. The physical meaning of individual terms in this equation is given in Sec. 2 . The last two terms on the RHS of Eq. (23) are comparable in magnitude. Indeed, by substituting $\mathbf{v}_{\perp}$ in the $F_{d i v}$ term with the $\nabla \mathrm{B}$ drift velocity $V_{\nabla B} \sim v_{i, t h} \times \rho_{i} / R$ (Eq. (A2)) and replacing the gradient sign with the inverse SOL width, $1 / \lambda_{\text {SOL }}$, (see discussion following Eq. (A2), noting that $V_{\nabla B}$ has a significant component perpendicular to the magnetic surface) one obtains the estimate $F_{d i v} \sim n m V_{\|} v_{i, t h} \times \rho_{i} /\left(\lambda_{S O L} R\right.$ ) for this term (a similar contribution comes from including $V_{E \times B}$ in $\mathbf{v}_{\perp}$, taking into consideration that $V_{E \times B}$ may be predominately in the poloidal direction). Turning to $F_{c u r v}$, since the poloidal $\mathrm{E} \times \mathrm{B}$ drift velocity is much greater than the poloidal $\nabla \mathrm{B}$ drift velocity, the latter can be neglected and, by estimating the absolute value of the curvature $\mathbf{k}$ as $1 / R$ and using $V_{E \times B, \theta} \sim v_{i, t h} \times \rho_{i} / \lambda_{S O L}$ (Eq. (A1)) for $\mathbf{v}_{\perp}$ in this term, one obtains the same estimate $n m V_{\|} v_{i, t h} \times \rho_{i} /\left(\lambda_{S O L} R\right)$ for $F_{\text {curv }}$.

Turning now to the term $-\left(n m V_{\|}^{2}+p_{\|}-p_{\perp}\right) \nabla_{\|} B / B$, which for the simplest case of isotropic ion pressure reduces to $-n m V_{\|}^{2} \nabla_{\|} B / B$. By equating $V_{\|}$to $V_{E, \|}$ (see Eq. (A4); we note, however, that experimental values of $V_{\|}>V_{E, \|}$ have been reported), and using $\nabla_{\|} B / B \sim \mathcal{E} / q R$, this term can be estimated, again, similar to the two above mentioned terms as: $n m V_{\|} v_{i, t h} \times \rho_{i} /\left(\lambda_{S O L} R\right)$. Hence, all the three terms can be roughly of the same order.

Comparison between the above drift-related terms and the main, pressure gradient term in Eq. (23), is difficult in the general case, as much depends on the poloidal distribution of plasma parameters and location ('main SOL' region, or the region near the divertor target). A maximum self-consistent estimate for the effect of drifts can, however, be made if one allows for very large pressure (and hence, electric potential) variations, of order of the pressure itself, over certain parallel distance $l_{\|}$(which does not necessarily coincide with $q R$ ). The parallel force due to the pressure gradient is then $\nabla_{\|} p \sim p / l_{\|}$. The radial velocity caused by the radial $\mathrm{E} \times \mathrm{B}$ drift is $\sim V_{E x B, r}=T_{e} / e B l_{\theta}$, where the poloidal length is related to the parallel length via $l_{\theta}=l_{\|} B_{\theta} / B$. The radial divergence of the momentum flux is $\sim n m_{i} V_{i \|} V_{r} / \lambda_{S O L}$. For an upper estimate for the ion parallel velocity of order the ion thermal velocity, $V_{i \|} \sim v_{i, t h}$, one can then obtain for the divergence of the momentum flux the estimate $F_{d r, \|} \sim p \rho_{i \theta} /\left(l_{\|} \lambda_{S O L}\right)$, where $\rho_{i \theta}$ is ion poloidal gyroradius. For the ratio of the two forces, one then obtains:

$$
\frac{F_{d r, \|}}{\nabla_{\|} p} \sim \frac{\rho_{i \theta}}{\lambda_{S O L}}
$$

A similar estimate was obtained for the maximum contribution of drifts to the particle flux (both parallel and perpendicular) in the SOL in [5], and it shows that the contribution of driftrelated terms to the parallel momentum balance equation can potentially be rather large.

We may follow the common procedure of introducing parallel viscous stress as a way of avoiding the presence of two pressures - $p_{\|}$and $p_{\perp}$ - in the momentum equation, with $(\vec{\nabla} \cdot \overrightarrow{\mathbf{P}})_{\|}$ 
given by Eq. (23). Unless this is done, two energy equations are required for closing the hierarchy of conservation equations, one for parallel energy and one for perpendicular energy, which is usually avoided for computational reasons. By introducing total pressure $p=\left(p_{\|}+2 p_{\perp}\right) / 3$, the term $\nabla_{\|} p_{\|}$in Eq. (23) can be replaced with the combination $\left(\nabla_{\|} p+\nabla_{\|} \Pi_{\|}\right)$, where $\Pi_{\|} \equiv\left(p_{\|}-p\right)=\frac{2}{3}\left(p_{\|}-p_{\perp}\right)$ is the parallel viscous stress. In order to close the equations, one then has to introduce an estimate for $\Pi_{\|}$in terms of already defined quantities, as will be discussed in Sec. 6. For weakly collisional conditions, which often occur in the SOL, however, the estimates for $\Pi_{\|}$are only very rough approximations and it can be more accurate to retain the two pressures, together with suitable approximations for the parallel and perpendicular energy equations. To date, SOL fluid modelling has not followed this approach but as hotter, less collisional SOL plasmas are encountered in more powerful devices, such as ITER, this may change.

We next consider the term $F_{d i v}$. By splitting particle velocity into random $\widetilde{\mathbf{v}}$ and mean $\mathbf{V}$ parts, so that $\langle\tilde{\mathbf{v}}\rangle=0$, this term can be written as:

$$
F_{\text {div }}=-\vec{\nabla} \cdot\left(n m V_{\|} \mathbf{V}_{\perp}\right)-\vec{\nabla} \cdot\left(n m\left\langle\tilde{v}_{\|} \tilde{\mathbf{v}}_{\perp}\right\rangle\right)
$$

The last term in this equation is the perpendicular viscous force $-\vec{\nabla} \cdot \vec{\Pi}_{\perp}$ and $\vec{\Pi}_{\perp}=n m\left\langle\tilde{v}_{\|} \tilde{\mathbf{v}}_{\perp}\right\rangle$ is the perpendicular viscous stress. In the literature of the field, the perpendicular viscosity term has proven to be one of the most difficult terms to analyse. It has a number of sources. In particular, perpendicular velocity $\mathbf{v}_{\perp}$ is affected by Coulomb collisions and the electromagnetic $Z e\left(\mathbf{E}+\mathbf{v}_{\perp} \times \mathbf{B}\right)$ force. The former is usually ignored although Braginskii gives estimates for the terms involved but the latter can be especially large in the presence of plasma turbulence which e.g. may result in large radial velocity due to $\mathbf{E}_{\theta} \times \mathbf{B}$ drifts caused by fluctuating poloidal electric fields - i.e. 'anomalous viscosity'.

One contribution to the viscosity comes from fast Larmor rotation (gyration) of particles with thermal velocities, in the quasi-stationary electromagnetic field which enters via products proportional to $v_{i \|} v_{i \perp, L}$. However, as noted earlier, it is possible to almost entirely eliminate the contribution of the Larmor rotation from the sum $-\left(F_{d i v}+F_{c u r v}\right)$ in the parallel momentum balance equation. The elimination of Larmor rotation is therefore general, covering both the convective as well as the viscous terms. Note however, that this elimination only applies to the momentum balance equation when formulated in terms of parallel and perpendicular pressures as independent variables. It does not apply to the formulation of this equation in terms of the total pressure and parallel viscous stress as the two independent variables, at least not when the standard approximations for $\Pi_{\|}$are used, since the latter does, in fact, include Larmor rotation contributions, see Sec. 6.

As noted in Section 2, the perpendicular velocity can be split into four parts, see Eq. (2). Owing to the elimination of the Larmor rotation from the parallel momentum equation, in both terms, convective, $\vec{\nabla} \cdot\left(n m V_{\|} \mathbf{V}_{\perp}\right)$, as well as viscous, $\vec{\nabla} \cdot\left(n m\left\langle\tilde{v}_{\|} \tilde{\mathbf{v}}_{\perp}\right\rangle\right)$, elimination of the 
' $L$ ' components allows one to replace $\mathbf{v}_{\perp}$ with $\mathbf{v}_{\perp, \text { g.c. }}$, where the guiding centre velocity can be defined as the sum of drift $\mathbf{v}_{\perp, d r}$ and anomalous $\mathbf{v}_{\perp, \text { anom }}$ velocities:

$$
\mathbf{v}_{\perp, \text { g.c. }}=\mathbf{v}_{\perp, d r}+\mathbf{v}_{\perp, \text { anom }}=\frac{\mathbf{E} \times \mathbf{B}}{B^{2}}+\frac{1}{2} \frac{m \bar{v}_{\perp}^{2}}{Z e B^{3}} \mathbf{B} \times \vec{\nabla} B+\frac{m \bar{v}_{\|}^{2}}{Z e B^{3}} \mathbf{B} \times(\mathbf{B} \cdot \nabla \mathbf{b})+\mathbf{v}_{\perp, \text { anom }}
$$

(The contribution of the velocity $\mathbf{v}_{\perp, \text { coll }}$ caused by the Coulomb collisions, to the momentum balance equation is usually neglected; as noted, this contribution, however, is included in Braginskii's collisional analysis). One can therefore express the term $F_{d i v}$ through parallel and guiding centre velocities as:

$$
F_{d i v}=-\vec{\nabla} \cdot\left(n m V_{\|} \mathbf{V}_{\perp, \text { g.c. }}\right)-\vec{\nabla} \cdot\left(n m\left\langle\tilde{v}_{\|} \tilde{\mathbf{v}}_{\perp, \text { g.c. }}\right\rangle\right)
$$

The second - viscous - term in $F_{d i v}$ requires further analysis, Sec. 5. The anomalous part $\tilde{\mathbf{v}}_{\perp \text {,anom }}$ of the guiding centre velocity $\widetilde{\mathbf{v}}_{\perp, \text { g.c. }}$ under most common conditions is a timedependent quantity, since it accounts for turbulent transport. Strictly speaking, therefore, one has to specify whether the whole expression given by Eq. (28) is to be evaluated at each particular instant or it is a time-averaged value. This cannot cause any problems when the anomalous velocity figures by itself as a term in an equation, as in the case of Eq. (27), since the result of the time-averaging is straightforward, and both $\mathbf{v}_{\perp, \text { g.c. }}$ and $\mathbf{v}_{\perp \text {,anom }}$ can be considered either as instantaneous or time-averaged quantities. In the products such as $\left\langle\tilde{v}_{\|} \tilde{\mathbf{v}}_{\perp, \text { g.c. }}\right\rangle$, however, the clear definition between the two possibilities must be made. We will be assuming that all such products are averages over time, but, in order not to complicate the expressions, will defer the explicit notations for the time-averaging until Sec. 5, when the products involving $\widetilde{\mathbf{v}}_{\perp, \text { anom }}$ specifically will be considered.

We now turn to the term $F_{\text {curv }}=n m\left\langle v_{\|} \mathbf{v}_{\perp}\right\rangle \cdot \mathbf{k}$ in Eq. (23). From the estimates made in App. A (related to the small value of curvature, $\sim 1 / R$ ), it is clear that only relatively large contributions to the perpendicular velocity $\mathbf{v}_{\perp}$, compared with the poloidal $\mathrm{E} \times \mathrm{B}$ drift velocity, need be included. Since $\nabla B$ and centrifugal drifts, as well as radial $E \times B$ drift, are much smaller than the poloidal $\mathrm{E} \times \mathrm{B}$ drift velocity, the latter is the only drift that needs to be included in $\mathbf{v}_{\perp}$. Anomalous/turbulent flux velocity $\mathbf{v}_{\perp \text {,anom }}$ is also typically smaller than the

E $\times \mathrm{B}$ drift velocity $\mathbf{V}_{E \times B}=\mathbf{E} \times \mathbf{B} / B^{2}$. Finally, the diamagnetic velocity is mainly caused by the superposition of Larmor rotations of individual particles, as was pointed out earlier, and, due to the elimination of the Larmor rotation from the terms containing products $v_{\|} \mathbf{v}_{\perp}$ in the parallel momentum balance equation, this velocity also is not to be included in the term $F_{\text {curv }}$. Therefore, it is sufficient to include only the $\mathrm{E} \times \mathrm{B}$ drift velocity in this term and, since this velocity is the same for all particles, no viscous contributions arise, so we can write:

$$
F_{\text {curv }}=n m\left\langle v_{\|} \mathbf{v}_{\perp}\right\rangle \cdot \mathbf{k} \approx n m V_{\|} \mathbf{V}_{E \times B} \cdot \mathbf{k} \approx-n m V_{\|} \mathbf{V}_{E \times B} \cdot \mathbf{R} / R^{2}
$$


In the particle balance equation of the system (1), by integrating Eq. (24) over the particle distribution and neglecting a small term $\frac{p_{\perp}}{e B} \mathbf{b}(\mathbf{b} \cdot$ curlb) (a discussion of this parallel drift will be presented in [42]), the perpendicular macroscopic drift flux is obtained:

$n \mathbf{V}_{\perp, d r}=\frac{n}{B^{2}} \mathbf{E} \times \mathbf{B}+\frac{p_{\perp}}{Z e B^{3}} \mathbf{B} \times \nabla B+\frac{\left(p_{\|}+n m V_{\|}^{2}\right)}{Z e B^{3}} \mathbf{B} \times \mathbf{B} \cdot \nabla \mathbf{b}$

Finally, $n \mathbf{V}=n \mathbf{V}_{\text {g.c. }}+\operatorname{curl} \mathbf{M} / Z e$ (see, e.g. [40, 1]). The last term here, being the curl of a vector, is automatically divergence-free. Hence one can replace $n \mathbf{V}$ with $n \mathbf{V}_{\text {g.c. }}=n \mathbf{V}_{\|}+n \mathbf{V}_{\perp, d r}+n \mathbf{V}_{\perp, \text { anom }}$ in the particle balance equation of the system (1) (also neglecting $\left.\mathbf{V}_{\perp, \text { coll }}\right)$.

The system (1), which, as noted, is applicable to each particle species, can now be written as:

$$
\begin{aligned}
& \left(\frac{\partial n}{\partial t}+\vec{\nabla} \cdot\left(n \mathbf{V}_{g . c .}\right)=S\right. \\
& \left\{\frac{\partial\left(n m V_{\|}\right)}{\partial t}+\nabla_{\|}\left(p_{\|}+n m V_{\|}^{2}\right)-\left(n m V_{\|}^{2}+p_{\|}-p_{\perp}\right) \nabla_{\|} B / B+\vec{\nabla} \cdot\left(n m V_{\|} \mathbf{V}_{\perp, g . c .}\right)+\right. \\
& \vec{\nabla} \cdot\left(n m\left\langle\tilde{v}_{\|} \tilde{\mathbf{v}}_{\perp, g . c .}\right\rangle\right)-n m V_{\|} \mathbf{V}_{E \times B} \cdot \mathbf{k}-e Z n E_{\|}=R_{\|}+F_{\|}
\end{aligned}
$$

Usually, the particle balance equation is only written for ions, with an addition of a separate equation for electric currents. The momentum balance equations for ions and electrons can, however, be summed together. This eliminates contributions from the parallel electric field and ion-electron friction forces. Also, since the ion mass is much greater than the electron mass, we can then neglect contributions from electrons in all terms except for the pressure terms, resulting in:

$$
\begin{array}{r}
\frac{\partial\left(n m_{i} V_{i \|}\right)}{\partial t}+\nabla_{\|}\left(p_{\|}+n m_{i} V_{i \|}^{2}\right)-\left(n m_{i} V_{\|}^{2}+p_{\|}-p_{\perp}\right) \nabla_{\|} B / B+\vec{\nabla} \cdot\left(n m_{i} V_{i \|} \mathbf{V}_{i \perp, g . c .}\right)+ \\
\vec{\nabla} \cdot\left(n m_{i}\left\langle\tilde{v}_{i \|} \tilde{\mathbf{v}}_{i \perp, \text { g.c. }}\right)\right)-n m_{i} V_{i \|} \mathbf{V}_{E \times B} \cdot \mathbf{k}=F_{\|}
\end{array}
$$

where parallel pressure $p_{\|}$and external force $F_{\|}$are sums of ion plus electron, parallel pressure and total external force. It may be noted that Eq. (32) is general, independent of the degree of collisionality.

Evaluation of the $F_{\text {div }}$ convective term $\vec{\nabla} \cdot\left(n m_{i} V_{i \|} \mathbf{V}_{i \perp \text {,g.c. })}\right.$ in Eq. (32) is straightforward, however, the $F_{\text {div }}$ viscous term $\vec{\nabla} \cdot\left(n m_{i}\left\langle\tilde{v}_{i \|} \tilde{\mathbf{v}}_{i \perp, \text { g.c. }}\right\rangle\right)$ requires further analysis, which we turn to next.

\section{Perpendicular convective and perpendicular viscous terms in the parallel momentum balance equation}


We now consider further the drift-term $F_{d i v}$ containing the average over the particle distribution, $n\left\langle\tilde{v}_{i \|} \widetilde{\mathbf{v}}_{i \perp, d r}\right\rangle$, which requires an estimate in terms of the basic fluid variables. As explained in the previous section, splitting particle velocity into random $\widetilde{\mathbf{v}}$ and mean $\mathbf{V}$ parts allows one to separate averages of the products $v_{\|} \mathbf{v}_{\perp}$ into mean, $\left\langle V_{\|} \mathbf{V}_{\perp}\right\rangle \equiv V_{\|} \mathbf{V}_{\perp}$, and random, $\left\langle\tilde{v}_{\|} \tilde{\mathbf{v}}_{\perp}\right\rangle$, parts. The contributions of the former to the momentum balance equation are called convective, the latter, viscous. Viscous contributions are always caused by deviations of the distribution function from Maxwellian, resulting in viscous stresses (described e.g. by vector $\vec{\Pi}$, for the parallel momentum equation). It is important to note, however, that viscous forces originate not only due to these stresses, but they also require spatial changes, being described by gradients, $-\vec{\nabla} \cdot \vec{\Pi}$.

We will follow below the standard procedure of splitting terms into convective and viscous, as they are often presented in equations for edge modelling. Some of these terms, e.g. anomalous viscous contributions, usually include coefficients which are not well-defined, see below.

The convective term $\vec{\nabla} \cdot\left(n m_{i} V_{i \|} \mathbf{V}_{i \perp \text {,g.c. }}\right)$ in Eq. (32) can be split into drift $\vec{\nabla} \cdot\left(n m_{i} V_{i \|} \mathbf{V}_{i \perp, d r}\right)$ and anomalous $\vec{\nabla} \cdot\left(n m_{i} V_{i \|} \mathbf{V}_{i \perp \text {,anom }}\right)$ components. The viscous term $\vec{\nabla} \cdot\left(n m_{i}\left\langle\tilde{v}_{i \|} \tilde{\mathbf{v}}_{i \perp, \text { g.c. }}\right\rangle\right)$ can also be split into drift $\vec{\nabla} \cdot\left(n m_{i}\left\langle\tilde{v}_{i \|} \tilde{\mathbf{v}}_{i \perp, d r}\right\rangle\right)$ and anomalous $\vec{\nabla} \cdot\left(n m_{i} \overline{\left\langle\tilde{v}_{i \|} \tilde{\mathbf{v}}_{i \perp, a n o m}\right\rangle}\right)$ components, where the bar $\overline{(\ldots)}$ denotes time-averaging. It turns out that the drift component of the viscous term contains a relatively large contribution from the ion centrifugal drift, comparable to the contribution of this drift to the convective term $\vec{\nabla} \cdot\left(n m_{i} V_{i \|} \mathbf{V}_{i \perp, d r}\right)$. This contribution must therefore be dealt with separately, and it can later be added to the convective drift term. The anomalous convective term includes the average ion velocity $\mathbf{V}_{i \perp \text {,anom }}$ which, unlike other quantities in this paper, has to be represented by double averaging, over the distribution function as well as time: $\mathbf{V}_{i \perp \text {,anom }}=\overline{\left\langle v_{i \perp, \text { anom }}\right\rangle}$, as it involves time-averaging of a fluctuating perpendicular velocity. The same applies to the anomalous viscous term.

The anomalous viscous term has to be represented with some empirical-based ansatz, involving an arbitrary coefficient of perpendicular viscosity, for example. The viscous drift term needs to be related to the basic fluid variables, such as pressure and heat flux density, and to the drift velocities. We will now derive the needed expressions for the viscous drift flux, $n\left\langle\tilde{v}_{i \|} \tilde{\mathbf{v}}_{i \perp, d r}\right\rangle$. It will be found that a particularly simple expression holds for the approximation of the actual velocity distribution by a drifting Maxwellian, Eq. (34). For the more realistic case it will be found necessary to add some further terms, Eq. (35), an approximation for which is given in Sec. 6.

From Eq. (24) for the ion component, by neglecting the first term on its RHS one obtains:

$$
n\left\langle\tilde{v}_{i \|} \tilde{\mathbf{v}}_{i \perp, d r}\right\rangle=\frac{1}{2} \frac{n m_{i}\left\langle\tilde{v}_{i \|} \bar{v}_{i \perp}^{2}\right\rangle}{Z e B^{3}} \mathbf{B} \times \nabla B+\frac{n m_{i}\left\langle\tilde{v}_{i \|} \bar{v}_{i \|}^{2}\right\rangle}{Z e B^{3}} \mathbf{B} \times(\mathbf{B} \cdot \nabla \mathbf{b})
$$


Since the particle parallel velocity is almost independent of the gyro-phase, $\bar{v}_{i \|}^{2}$ can be replaced with $v_{i \|}^{2}$. Note that the $\mathrm{E} \times \mathrm{B}$ drift doesn't contribute to the above equation since it has a constant value for all particles (i.e. there is no dependence on $v_{i \|}$ or $v_{i \perp}$ ), resulting in $\left\langle\tilde{v}_{i \|} \mathbf{V}_{E \times B}\right\rangle=0$ (generally, one can add any constant to the second component of the averaged product of $\tilde{v}_{i \|}$ with that component without changing the result; for this reason, the full expression for ion $\nabla \mathrm{B}$ and centrifugal drifts is used in Eq. (33), as there is no need to calculate their random part(s)). In the first term on the RHS of this equation, $\bar{v}_{i \perp}$ can be replaced with the perpendicular velocity of an individual Larmor circle: $\bar{v}_{i \perp}^{2} \approx v_{i \perp, L}^{2}$. For the approximation given by a drifting ion Maxwellian distribution function (the more realistic case is considered below) with mean parallel velocity $V_{i \|},\left\langle\tilde{v}_{i \|} \bar{v}_{i \perp}^{2}\right\rangle=0$, and the first term on the RHS of Eq. (33) disappears. Also $\left\langle\tilde{v}_{i \|} v_{i \|}^{2}\right\rangle=2 V_{i \|}\left\langle\tilde{v}_{i \|}^{2}\right\rangle$, and we have taken into account that $\left\langle\tilde{v}_{i \|} V_{i \|}^{2}\right\rangle=\left\langle\tilde{v}_{i \|}^{3}\right\rangle=0$. By replacing $n m_{i}\left\langle\tilde{v}_{i \|}^{2}\right\rangle$ with $p_{i \|}$, the last term in Eq. (33) can be expressed in the 'convective' form as $n V_{i \|} \mathbf{V}_{i \perp, p \|}^{*}$, where we define:

$$
n \mathbf{V}_{i \perp, p \|}^{*}=\frac{2 p_{i \|}}{Z e B^{3}} \mathbf{B} \times \mathbf{B} \cdot \nabla \mathbf{b}
$$

Therefore, for the special (approximate) case of a drifting ion Maxwellian distribution function the viscous flux $n\left\langle\tilde{v}_{i \|} \tilde{\mathbf{v}}_{i \perp, d r}\right\rangle$ is reduced to $n V_{i \|} \mathbf{V}_{i \perp, p \|}^{*}$. Comparing this flux with the expression for the part of the convective flux $n V_{i \|} \mathbf{V}_{i \perp, d r}$ associated with the ion centrifugal drift (by replacing $n \mathbf{V}_{i \perp, d r}$ with $\frac{\left(p_{\|}+n m V_{\|}^{2}\right)}{Z e B^{3}} \mathbf{B} \times \mathbf{B} \cdot \nabla \mathbf{b}$, as follows from Eq. (30)), one concludes that the contribution of the ion centrifugal drift to the viscous term $\vec{\nabla} \cdot\left(n m_{i}\left\langle\tilde{v}_{i \|} \tilde{\mathbf{v}}_{i \perp, d r}\right\rangle\right)$ (which corresponds to the last term of Eq. (33)) is significant: it is almost twice as large as its contribution to the convective term $\vec{\nabla} \cdot\left(n m_{i} V_{i \|} \mathbf{V}_{i \perp, d r}\right)$ under (quite typical) conditions when the ion parallel convective energy flux $n m_{i} V_{i \|}^{2}$ is much less than the parallel ion pressure.

Since one of the main points in this section is the interpretation of the role of the centrifugal drift, for the sake of clarity it is useful here to present a compact expansion in parallel velocity that results in both convective and viscous forces. The contribution of each individual particle to the parallel momentum transport related by this drift is proportional to $v_{\|}^{3}$, where $v_{\|}$is the parallel velocity of the particle. Splitting it into average (for a particular species) and the deviation from this average (which can also be called 'chaotic'), expanding and then averaging over the distribution function, one obtains: $\left\langle v_{\|}^{3}\right\rangle=\left\langle\left(V_{\|}+\tilde{v}_{\|}\right)^{3}\right\rangle=$ $\left\langle V_{\|}^{3}+3 V_{\|}^{2} \tilde{v}_{\|}+3 V_{\|} \tilde{v}_{\|}^{2}+\tilde{v}_{\|}^{3}\right\rangle$. The $1^{\text {st }}$ and part of the $3^{\text {rd }}$ term, namely $\left\langle V_{\|} \tilde{v}_{\|}^{2}\right\rangle$, on the RHS correspond to the convective momentum transport, while $\left\langle 2 V_{\|} \tilde{v}_{\|}^{2}\right\rangle$ and the last term - to the 
viscous contribution. The $2^{\text {nd }}$ term is equal to zero, while the last term is zero only for a Maxwellian distribution. It will be considered in more detail below.

Of course, the existence of a (drifting) Maxwellian distribution implies extremely strong collisionality, which would also result in $p_{\|}=p_{\perp}=p$ (and $\Pi_{\|}=0$ ). Thus we can use $p_{i}$ in Eq. (34) in the case of extremely high collisionality. We will, however, retain the version of Eq. (34) that depends on $p_{\|}$for the general case, considered next.

In the general case of an arbitrary (i.e. not necessarily drifting Maxwellian) and more realistic ion distribution function, a viscous term $\vec{\nabla} \cdot \vec{\Pi}_{i \perp, \nabla B+c e n t}$ has to be added to the LHS of Eq. (32), where $\vec{\Pi}_{i \perp, \nabla B+c e n t}$ is defined by:

$$
\vec{\Pi}_{i \perp, \nabla B+c e n t}=\left\langle\tilde{v}_{i \|} v_{i \perp}^{2}\right\rangle \frac{n m_{i}^{2}}{2 Z e B^{3}} \mathbf{B} \times \nabla B+\left\langle\tilde{v}_{i \|}^{3}\right\rangle \frac{n m_{i}^{2}}{Z e B^{3}} \mathbf{B} \times(\mathbf{B} \cdot \nabla \mathbf{b})
$$

We need to relate the two averages over the velocity distribution, $\left\langle\tilde{v}_{i \|} \bar{v}_{i \perp}^{2}\right\rangle$ and $\left\langle\tilde{v}_{i \|}^{3}\right\rangle$, to the basic fluid variables, which is done in Sec 6, using estimates obtained in the closure procedure. Here we obtain a first rough estimate by taking $\left\langle\tilde{v}_{i \|} \bar{v}_{i \perp}^{2}\right\rangle \sim\left\langle\tilde{v}_{i \|}^{3}\right\rangle \sim V_{i \mid} p_{i}$; a better estimate in terms of ion parallel heat flux density, $q_{i \|}$, will be given in Sec. 6. Also, in most cases the difference between $\mathbf{B} \times \nabla B$ and $\mathbf{B} \times \mathbf{B} \cdot \nabla \mathbf{b}$ vectors can also be neglected, as both are approximately equal to $\vec{e}_{v e r t} B^{2} / R$ with the unit vector $\vec{e}_{v e r t}$ pointing vertically in the direction of the ion $\nabla \mathrm{B}$ drift. The convective particle flux due to the sum of the ion $\nabla \mathrm{B}$ and centrifugal drifts can then be expressed as $n \mathbf{V}_{i \perp, \nabla B+c e n t}=\frac{2 p_{i}}{Z e B R} \vec{e}_{v e r t}$. Under these conditions, the parallel momentum transport described by the combined convective term $\vec{\nabla} \cdot\left(n m_{i} V_{i \|} \mathbf{V}_{i \perp, \nabla B+c e n t}\right)$ is effectively doubled due to the contribution made by the ion centrifugal drift to the viscous term $\vec{\nabla} \cdot\left(n m_{i}\left\langle\tilde{v}_{i \|} \tilde{\mathbf{v}}_{i \perp, d r}\right\rangle\right)$.

Using the above results, and by splitting both convective and viscous momentum transport terms into the drift and anomalous contributions (see above), the system of particle and parallel momentum balance equations given by Eq. (31) can be finally cast into the following form:

$$
\left\{\begin{aligned}
& \frac{\partial n}{\partial t}+\vec{\nabla} \cdot\left(n \mathbf{V}_{i \|}+n \mathbf{V}_{i \perp, d r}+n \mathbf{V}_{i \perp, a n o m}\right)=S \\
& \frac{\partial\left(n m_{i} V_{i \|}\right)}{\partial t}+ \nabla_{\|}\left(p_{\|}+n m_{i} V_{i \|}^{2}\right)-\left(n m_{i} V_{i \|}^{2}+p_{\|}-p_{\perp}\right) \nabla_{\|} B / B+\vec{\nabla} \cdot\left(n m_{i} V_{i \|}\left(\mathbf{V}_{i \perp, d r}+\mathbf{V}_{i \perp, a n o m}\right)\right)+ \\
& \vec{\nabla} \cdot\left(n m_{i} V_{i \|} \mathbf{V}_{i \perp, p \|}^{*}\right)+\vec{\nabla} \cdot \vec{\Pi}_{i \perp, \nabla B+c e n t}+\vec{\nabla} \cdot \vec{\Pi}_{i \perp, a n o m}-n m_{i} V_{\|} \mathbf{V}_{E \times B} \cdot \mathbf{k}=F_{\|}
\end{aligned}\right.
$$


In this system of equations $p$ denotes the sum, ion plus electron, pressure, $n \mathbf{V}_{i \perp, d r}$ is ion perpendicular drift flux given by Eq. (30), $n \mathbf{V}_{i \perp \text {,anom }}$ is ion perpendicular anomalous flux, $n \mathbf{V}_{i \perp, p \|}^{*}$ is given by Eq. (34), $\vec{\Pi}_{i \perp, \nabla B+c e n t}$ is the ion perpendicular viscous stress caused by viscous effects given by Eq. (35) in the perpendicular momentum transport caused by the ion $\nabla \mathrm{B}$ and centrifugal drifts: namely, by the deviation of the ion distribution function from the drifting Maxwellian (for the latter, both terms on the RHS of Eq. (35) are zero, as stated earlier);

$$
\vec{\Pi}_{i \perp, \text { anom }}=n m_{i}\left\langle\overline{\tilde{v}_{i \|} \tilde{\mathbf{v}}_{i \perp, \text { anom }}}\right\rangle
$$

is the ion perpendicular anomalous viscous stress. Finally, $F_{\|}$stands for all other forces, including forces arising from interaction with neutrals.

In the numerical modelling, anomalous viscosity is usually introduced via an anomalous perpendicular viscosity diffusion coefficient $\eta_{i \perp, a n o m}$, which in turn is often related to the anomalous perpendicular particle diffusion coefficient by using a constant numerical coefficient $\alpha$ of order unity, so that $\eta_{i \perp, \text { anom }}=\alpha \mathrm{nm}_{i} D_{i \perp, \text { anom. }}$. In a simplest rectangular geometry, this results in $\vec{\Pi}_{i \perp \text {,anom }}=-\eta_{i \perp, \text { anom }} \partial V_{i \|} / \partial s_{\perp}$ and a parallel viscous force $-\partial \vec{\Pi}_{i \perp, a n o m} / \partial s_{\perp}$. The anomalous perpendicular particle flux will then be equal to $\Gamma_{\perp, \text { anom }}=-D_{i \perp, \text { anom }} \partial n / \partial s_{\perp}$, creating convective momentum flux $m_{i} \Gamma_{\perp, \text { anom }} V_{i \|}$ and the corresponding parallel force $-\partial\left(m_{i} \Gamma_{\perp, \text { anom }} V_{i \|}\right) / \partial s_{\perp}$. The ion perpendicular anomalous viscous stress is generally non-zero, in particular because the phasing of $\tilde{v}_{i \|}$ and $\tilde{\mathbf{v}}_{i \perp \text {,anom }}$ fluctuations doesn't have to be orthogonal. The part of the non-zero viscous stress $\vec{\Pi}_{i \perp \text {,anom }}$ attributed to time-averaging is known as the Reynolds stress. According to [48], this stress, by way of a corresponding phasing of the two velocity perturbations, can make a significant contribution to the parallel momentum balance equation.

The anomalous perpendicular particle flux is equal to $\Gamma_{\perp, \text { anom }}=-D_{i \perp, \text { anom }} \partial n / \partial s_{\perp}$, creating convective momentum flux $m_{i} \Gamma_{\perp, a n o m} V_{i \|}$ and the corresponding parallel force $-\partial\left(m_{i} \Gamma_{\perp, \text { anom }} V_{i \|}\right) / \partial s_{\perp}$. As noted above, a proper calculation of the perpendicular viscous stress $\vec{\Pi}_{i \perp, \nabla B+c e n t}$ requires knowledge of the averages $\left\langle\tilde{v}_{i \|} \bar{v}_{i \perp}^{2}\right\rangle$ and $\left\langle\tilde{v}_{i \|}^{3}\right\rangle$. This is addressed in Sec. 6 as part of the process of closing the set of equations.

Finally, we may note that Eq. (36) is still explicitly independent of the degree of collisionality; however, there are a number of quantities which are averages over the velocity distribution - namely $p_{\|}=n m\left(\left\langle v_{\|}^{2}\right\rangle-V_{\|}^{2}\right), \quad p_{\perp}=n m\left\langle v_{\perp}^{2}\right\rangle / 2, \quad\left\langle\tilde{v}_{i \|} v_{i \perp}^{2}\right\rangle$ and $\left\langle\tilde{v}_{i \|}^{3}\right\rangle$. The treatment of these quantities is governed by the process of closing the set of fluid equations, to which we turn next, Sec. 6. In one approach - appropriate for strongly collisional conditions - estimates are made for these 4 quantities in terms of the basic fluid variables, $n$, $\mathbf{V}, p$ (or $T$ ) and their gradients. For weakly collisional conditions it can be appropriate to employ two energy equations, for $p_{\perp}$ and $p_{\|}$, together with assumptions such as the neglect of $\left\langle\tilde{v}_{i \|}^{3}\right\rangle$. 


\section{Closing the Fluid Equations. Collisionality. Parallel Viscosity.}

While this paper only deals explicitly with the first two fluid conservation equations, it is not possible to entirely avoid consideration of the third - energy - equation because of the important issue of how to close the set of fluid equations. Closing the set of equations in turn is intimately related to the degree of collisionality which, in turn, dictates how to deal with the presence of two different pressures in the momentum equation - $p_{\perp}$ and $p_{\|}-$and the question of when it is appropriate to replace them with a single pressure by introducing $\Pi_{\|}$. The choice between using the two individual pressures or introducing the parallel stress is one of the principal issues involved in closing the equation set.

The simplest case is the limit of infinitely strong collisionality, which makes the velocity distribution a (perhaps drifting) Maxwellian, and thus $p_{\|}=p_{\perp}=p=n T$ (thus $\Pi_{\|}=0$ ), also $\left\langle\tilde{v}_{i \|} v_{i \perp}^{2}\right\rangle=0$, and $\left\langle\tilde{v}_{i \|} v_{i \|}^{2}\right\rangle=2 V_{i \|}\left\langle\tilde{v}_{i \|}^{2}\right\rangle=2 V_{i \|} p_{i} / n m_{i}$.

At the other extreme - for weak collisionality - as noted above, it is appropriate to retain the two pressures in the momentum equation, necessitating two energy equations. Closure is then dealt with by invoking various assumptions in the energy equations such as the neglect of parallel heat conduction, see discussion in [44] and also below.

For strong collisionality it is appropriate and convenient to work with $p$ and $\Pi_{\| \text {, which }}$ requires an estimate for $\Pi_{\|}$in terms of the basic fluid variables and their gradients. In order to close the equation set, similar estimates can be made for the other non-basic variables appearing in the momentum and energy equation, such as heat conduction; first estimates for these transport coefficients can be made using simple kinetic theory. Better estimates can be obtained using the Chapman and Cowling, Spitzer, Braginskii, etc. small perturbation method.

The focus of the present paper is on the effect of drifts, arbitrary magnetic geometry and Larmor rotation. We do not attempt here to make any original contribution to the methods for estimating $\Pi_{\|}$. For completeness, however, we now briefly review some of the results in the literature.

Before addressing the main issue - the ion $\Pi_{\|}$- we will first consider $\Pi_{\mathrm{e} \|}$. The contribution from electrons to $\Pi_{\|} \equiv \Pi_{i \|}+\Pi_{e \|}$ (note however that we often omit the subscript ' $i$ ' in $\Pi_{i \|}$ in this paper where it cannot cause confusion) is usually ignored due to their much higher collision rates compared to the ions, as was stated earlier. Further, in strongly collisional plasmas maxwellianization of both ions and electrons is rather strong, and any correction even to the ion (drifting) Maxwellian distribution due to e.g. the presence of parallel ion heat flux, comes only as first order corrections in the expansion in the ratio of the ion mean free path to the parallel system size (or characteristic parallel length describing changes of the plasma parameters), $\lambda_{i i} / L_{\|}$. Quite often, however, modelling has to be extended to plasmas which, in certain domains of the computational grid, are much less collisional, with $\lambda_{i i}$ approaching, or sometimes even exceeding $L_{\|}$. Under these circumstances, rather than abandoning altogether modelling attempts using collisional codes, it is often possible to 
extend the collisional model by including kinetic effects via the introduction of various flux limits (see e.g. [1 (p.656), 44, 49]). Such limits are typically introduced for ion and electron parallel heat flows and for the ion parallel viscosity. In extreme cases it is also possible that electron kinetic effects can become so strong that electron parallel and perpendicular chaotic energies can be different resulting in a difference between $p_{e \perp}$ and $p_{e \|}$. Therefore, for generality, we will retain terms with $\Pi_{e \|}$ in the equation, even though under typical conditions electron parallel viscous stress can be neglected, as stated above.

Turning now to the main closure issue, $\Pi_{i \|}$ : the method employed by Zawaideh et al [44] for estimating $\Pi_{i||}$ is particularly simple and illuminating. We start from the simplest-case version of their two energy equations for $p_{\perp}$ and $p_{\|}$:

$$
\begin{aligned}
& V_{i \|} \frac{d p_{i \|}}{d s}+3 p_{i \|} \frac{d V_{i \|}}{d s}=Q_{i, \perp \rightarrow \|}=\frac{2\left(n k T_{i \perp}-n k T_{i \|}\right)}{\tau_{i i}}=\frac{2\left(p_{i \perp}-p_{i \|}\right)}{\tau_{i i}}=\frac{-3 \Pi_{i \|}}{\tau_{i i}} \\
& V_{i \|} \frac{d p_{i \perp}}{d s}+p_{i \perp} \frac{d V_{i \|}}{d s}=Q_{i, \| \rightarrow \perp}=-\frac{\left(n k T_{i \perp}-n k T_{i \|}\right)}{\tau_{i i}}=-\frac{\left(p_{i \perp}-p_{i \|}\right)}{\tau_{i i}}=\frac{3 \Pi_{i \|}}{2 \tau_{i i}}
\end{aligned}
$$

assuming steady-state, $1 \mathrm{D}$, constant and straight $\mathbf{B}$, no sources, no heat conduction, and no ei heat transfer. The ion-ion collision time in the above equations is the pressure anisotropy relaxation time $\tau_{i i}=\frac{15 \sqrt{m_{i}} T_{\text {eff }}^{3 / 2}}{8 \sqrt{\pi} n_{i} e^{4} \ln \Lambda}$ used in [44]. The factor 2 in the definition of the energy transfer term $Q_{i, \perp \rightarrow \|}$ reflects the two degrees of freedom in the $\perp$-direction compared with one in the $\|$-direction. Subtracting these two equations and neglecting terms involving derivatives of $\Pi_{i \|}$ and the product of $\Pi_{i \|}$ with $d V_{i \|} / d s$ as being small - as implied by the strong collisionality assumption of small $\lambda / L$ which makes all gradients, as well as $\Pi_{i \|}$, small - one obtains:

$$
\Pi_{i \|}=-\frac{4}{9} \tau_{i i} p_{i} \frac{d V_{i \|}}{d s}
$$

This is identical with the lead term in Braginskii's estimate, considered next. Zawaideh's approach [44] makes the basic physical concept underlying $\Pi_{\|}$particularly clear: acceleration of a flow tends to cool it - reducing $T_{\|}$in the first instance as parallel random energy is converted to parallel flow energy. This tends to cause $T_{\|}$and $T_{\perp}$ to diverge, i.e. for $\left|\Pi_{\|}\right|$to increase, an effect which is opposed by the collisional heat transfer between the $\perp$ and $\|$ 'heat reservoirs'. Thus $\left|\Pi_{\|}\right| \mid$increases with $\left|d V_{i \mid} / \mathrm{d} s\right|$ and with $\tau_{i i}$. Although not included in the results of [44] or [40], one can readily show that other processes, e.g. heat transport, can also cause $T_{\|}$and $T_{\perp}$ to diverge. For example, it is readily shown that including the contributions of heat conduction to the two energy equations adds terms $2 \frac{d q_{i \|}^{\|}}{d s}$ and $\frac{d q_{\mathrm{i} \|}^{\perp}}{d s}$ to the RHS of Eqs. (38) and (39) respectively, where $q_{i \|}^{\|} \equiv \int f_{i} \frac{m_{i} v_{\|}^{2}}{2} v_{\|} d \mathbf{v}$ and $q_{i \|}^{\perp} \equiv \int f_{i} \frac{m_{i} v_{\perp}^{2}}{2} v_{\|} d \mathbf{v}$ are components of 
the parallel ion heat flux density $q_{i \|} \equiv \int f_{i} \frac{m_{i} v^{2}}{2} v_{\|} d \mathbf{v}$. This then adds a component to $\Pi_{i \|}$ of $-\frac{2}{9} \tau_{i i} \frac{d\left(2 q_{i \|}^{\|}-q_{i \|}^{\perp}\right)}{d s} \approx-\frac{2}{9} \tau_{i i} \frac{d q_{i \|}}{d s}$. A collision-based transport coefficient estimate for $q_{i \|}$ is then needed to close the equations. From simple kinetic theory one obtains $q_{i \|} \sim-\left(n T_{i} \tau_{i i} / m_{i}\right) \nabla_{\|} T_{i}$. An improved estimate is obtained from the perturbation approach of Chapman and Cowling, Spitzer, Braginskii, etc., of $q_{i \|}=-3.9 \frac{n T_{i} \tau_{i}}{m_{i}} \nabla_{\|} T_{i}$, where $\tau_{i}$ is the angle scattering ion-ion collisional time given by $\tau_{i}=\frac{3 \sqrt{m_{i}} T_{i}^{3 / 2}}{4 \sqrt{\pi} \lambda e^{4} Z_{i}^{4} n_{i}}$. Recently Fundamenski [50] has further generalized Eqs. $(38,39)$, resulting in more complete expressions for $\Pi_{i \|}$, and explicitly bringing out the transition from strong to weak collisionality.

This method of estimating $\Pi_{\|}$also makes clear the effect of the transition from strong to weak collisionality and also indicates the need for placing a 'kinetic limit' factor on any estimate, such as Eq. (40), obtained assuming strong collisionality: For very low collisionalities, as $\tau_{i} d V_{i \|} / d s \rightarrow \infty$, then $\left|\Pi_{\|}\right| \rightarrow \infty$ also, which is unphysical since $\Pi_{\|}=p_{\|}-p$, and so at the extreme where $p_{\|} \rightarrow 0, \Pi_{\|} \rightarrow-p$, i.e. $\left|\Pi_{\|}\right|$cannot exceed $p$. More detailed considerations indicate a limit of about $-1 / 2 p$ for $\Pi_{\|}[49]$. At the other extreme, where $p_{\|}=p_{\perp}, \Pi_{\|}=0$, of course. Similarly a kinetic correction factor can be applied to the above collisional transport coefficients for $q_{\|}$, see discussion in Chap. 26 of [1] (see also [50]).

We quote next Braginskii's well-known estimate for the parallel ion viscous stress which has been frequently used in theories and numerical codes, as given by his Chapman and Cowling type transport-coefficient, small-perturbation analysis, appropriate in the strong collisionality limit, and where he also allows for 3D ion fluid velocity [40]:

$$
\Pi_{i \| \text { Brag }}=-\eta_{o}^{i}\left(2 \nabla_{\|} V_{i \|}-(2 / 3) \operatorname{div} \mathbf{V}_{i}\right)
$$

where $\eta_{o}^{i}=0.96 n_{i} T_{i} \tau_{i}$ is the parallel ion viscosity coefficient (note that the ion-ion collision time $\tau_{i}$ is used). The absence of plasma (ion) density multiplier inside the divergence operator (which would have otherwise created particle flux $n \mathbf{V}_{i}$, in which the contribution of the Larmor rotation, described by the curl of a vector, could then be immediately eliminated) implies that the Larmor rotation contribution cannot be eliminated from the parallel viscous force. We note here that such an elimination does however occur if, instead of using full ion pressure inside the term $\nabla_{\|}\left(p+n m_{i} V_{i \|}^{2}\right)$, one uses parallel pressure $p_{\|}$, as in the original expression for the parallel component of the gradient of the stress tensor Eq. (23) and in Eqs. $(31,32,36)$, since $\operatorname{div} \boldsymbol{V}_{i}$ does not appear in any expression. That is, the replacement of $p_{/ /}$and $p_{\perp}$ with $p$ and $\Pi_{/ /}$modifies the otherwise simple result that Larmor rotation contributions are absent from the particle and momentum equations.

It can be readily shown that the contribution of Larmor rotation, i.e. of the ion diamagnetic velocity, to $\operatorname{div} \boldsymbol{V}_{i}$ is generally significant compared to $\nabla_{/ /} V_{i, / /}$, by considering the poloidal divergence of the ion diamagnetic velocity $\mathbf{B} \times \vec{\nabla} p_{i} / e n B^{2}$ owing to the $B$ - and $R$-variation. 
Pfirsch-Schlüter flow is a result of the divergence of poloidal diamagnetic and $\mathrm{E} \times \mathrm{B}$ flows. The latter two are roughly the same, so we can expect parallel divergence of the PfirschSchlüter flow (particle flux density) to be similar to the poloidal divergence of the ion diamagnetic flow. We also know that the plasma density tends to be fairly constant poloidally in the 'main SOL', due to the tendency of the plasma pressure to reach equilibrium and relatively poloidally constant $T_{e}, T_{i}$. Hence, the above conclusion can also be applied to divergences of ion poloidal and parallel velocities.

In the models and numerical codes where drift contributions are not included, the contribution of the perpendicular ion flux in Eq. (41) is often ignored, and only the parallel ion velocity is left (although, as just shown, it is arguable that this can be justified in most experimental conditions, where perpendicular drift and anomalous flux usually play an important role in $\operatorname{div} \mathbf{V}_{i}$ ). The expression for the parallel viscous stress given by Eq. (41) can then be simplified and adapted for an arbitrary magnetic geometry. Let's assume a parallel plasma flow inside a flux tube of a variable cross-section. For parallel velocities $V_{1}$ and $V_{2}$ (with $V_{1} \approx V_{2} \approx V$ ) at the ends of a flux tube with cross-sections $S_{1}$ and $S_{2}$ (where $S_{1} \approx S_{2} \approx \mathrm{S}$ ) and with a small distance between the tube ends $\Delta l_{\|}$, $\operatorname{div} \mathbf{V}$ can be expressed as $\left(V_{2} S_{2}-V_{1} S_{1}\right) /\left(\Delta l_{\|} S\right)$, or, in a differential form, as $\left(\nabla_{\|} V+V \nabla_{\|} S / S\right)$. Since the cross-section of the flux tube is inversely proportional to the magnetic field $B, \operatorname{div} \mathbf{V}=\left(\nabla_{\|} V-V \nabla_{\|} B / B\right)$. Hence, we arrive at the following expression:

$$
\Pi_{i \|}=-\frac{4}{3} \eta_{o}^{i}\left(\nabla_{\|} V_{i \|}+V_{i \|} \frac{\nabla_{\|} B}{2 B}\right)
$$

This equation is often used in numerical codes which don't include drifts. It coincides with Eq. (50) of [44], apart from a slightly different numerical coefficient $\left(-(4 / 9) p_{i} \tau_{i i}\right.$, Eq. (42), but note the difference between the ion-ion relaxation times, with $\tau_{i i}$ being larger by a factor of 2.5 than $\tau_{i}$ ). Thus, as noted earlier, the main part of Braginskii's estimate for $\Pi_{\| i}$ coincides with the high collisionality limit of Zawaideh's estimate [44] - even including the main part of the effect of a spatially varying field.

The expression Eq. (41) has usually been taken to be the largest contribution to the parallel ion viscous stress within the framework of the Chapman and Cowling (Braginskii) approach for transport equations of a strongly collisional plasma. This corresponds to a situation where the principal effect causing $T_{\|}$(thus $p_{\|}$) to diverge from $T_{\perp}$ (thus $p_{\perp}$ ) along the parallel direction, is the cooling caused by acceleration of the flow, which is $\propto \nabla_{\|} V_{\|}$. As already noted, in principle, many other effects, such as parallel gradients of parallel heat flux, can also cause $p_{\|}$and $p_{\perp}$ to diverge. In all estimates, the Chapman and Cowling type of formulation assumes strong (of order of $100 \%$ ) variation of plasma parameters along the field lines over the collisional mean free path. Under these conditions, other viscous contributions, e.g. those arising from the parallel gradients of parallel ion heat flux, $\sim \tau_{i} \nabla_{\|} q_{i \|}$ (see below), are only $\sim \lambda_{i i} / L_{\|}$of the main term $\sim \eta_{o}^{i} \nabla_{\|} V_{i \|}$ given by Eq. (41) and therefore can be neglected. It was later realized, however [41], that such an assumption is not adequate for a range of experimental conditions where some special relations between perturbations of various plasma parameters occur. Consider, for example, the case of parallel ion particle and heat fluxes arising in the main tokamak SOL (far away from the divertor) due to the 
divergence of corresponding poloidal particle and heat fluxes of order $V_{i \theta} \approx \nabla_{r} p_{i} / e B$ and $q_{i \theta} \sim p_{i} \nabla_{r} T_{i} / e B$, respectively. Assuming $\nabla_{r} p_{i} \sim n \nabla_{r} T_{i}$, these particle and heat fluxes will be related as $q_{i \theta} \sim V_{i \theta} p_{i}$. Since both vary by $\sim \mathcal{E}$ over the flux surface, their poloidal divergencies result in parallel fluxes of amplitudes (and changing signs over $\sim q R$ ) of $q_{i \|} \sim V_{i \|} p_{i}$. The two contributions to the parallel ion viscous stress will then become comparable: $\tau_{i} \nabla_{\|} q_{i \|} \sim \eta_{o}^{i} \nabla_{\|} V_{i \|}[41]$.

Recently, further advance in the Chapman and Cowling type of derivation of the parallel ion viscous stress has been made by including contributions that arise from the full nonlinear form of the collision operator, giving rise to terms proportional to the square of the heat flux which can be of the same order as terms $\sim \tau_{i i} \nabla_{\|} q_{i \|}$ [51]. The expression for the parallel ion viscous stress that follows from [51] (see p.95 of this Ref.) is quoted next:

$$
\begin{gathered}
\Pi_{i \|}=-0.0426 \frac{m_{i} q_{i}^{2}}{T_{i} p_{i}}+0.0993 \frac{m_{i} q_{i \|}^{2}}{T_{i} p_{i}}+0.6398 \frac{p_{i}}{v_{i}}\left(\vec{\nabla} \cdot \mathbf{V}_{i}-3 \mathbf{b} \cdot \nabla_{\|} \mathbf{V}_{i}\right)-\frac{0.7131}{T_{i} v_{i}} \mathbf{q}_{i \|} \cdot \vec{\nabla} T_{i}+ \\
\frac{0.4133}{v_{i}}\left(\vec{\nabla} \cdot \mathbf{q}_{i}-3 \mathbf{b} \cdot \nabla_{\|} \mathbf{q}_{i}\right)+\frac{0.0419}{v_{i}}\left(\vec{\nabla} \cdot \mathbf{q}_{i \|}-3 \mathbf{b} \cdot \nabla_{\|} \mathbf{q}_{i \|}\right)-\frac{0.1573}{p_{i} v_{i}}\left(\mathbf{q}_{i} \cdot \vec{\nabla} p_{i}-3 \mathbf{q}_{i \|} \cdot \vec{\nabla} p_{i}\right)
\end{gathered}
$$

Here $\mathbf{q}_{i}$ and $\mathbf{q}_{i \|}$ are full and parallel ion heat fluxes, respectively, $v_{i}$ is the inverse Braginskii ion-ion collision time, $1 / \tau_{i}$. The $3^{\text {rd }}$ term on the RHS of the above equation corresponds to Braginskii's viscous stress, and it matches Eq. (42) for the case of simple rectangular magnetic geometry. This term, however, as well as the whole expression given by Eq. (43), applies for an arbitrary curvilinear magnetic geometry. When $\mathbf{q}_{i} \sim \mathbf{q}_{i \|}$ and $\vec{\nabla} \cdot \rightarrow \nabla_{\|}$the sum of the $5^{\text {th }}$ and $6^{\text {th }}$ terms in Eq. (43), proportional to the gradient of the heat flux density, are within $\sim 20 \%$ of the simple estimate above. All terms in Eq. (43) (except, perhaps, for the $6^{\text {th }}$ term on the RHS, which may be numerically small) can be of the same order and should be kept in this equation, as was pointed out in [51].

The pressure difference term $-\left(p_{\|}-p_{\perp}\right) \nabla_{\|} B / B$ in the momentum balance equation Eq. (36) for ions directly follows from Eq. (43) and $\left(p_{i \|}-p_{i \perp}\right)=3 \Pi_{i \|} / 2$.

As noted above, when employing the standard strong-collisionality approach it is necessary to include kinetic flux limiters in any expression for $\Pi_{\|}$, even for refined estimates such as Eq. (43), in order to prevent $\nabla_{\|} \Pi_{\|}$from becoming unphysically large when $\lambda_{i i} / \mathrm{L}_{\|}$is large $[49,1,51]$. Such kinetic correction factors are, however, only rough estimates. One can also obtain approximations for $\Pi_{i / /}$ using two-pressure $\left(p_{i \perp}, p_{i \|}\right)$ formulations, such as Eqs. (38, 39), but this requires making simplifications for the energy equations for $p_{i \perp}$ and $p_{i \|}$, such as neglecting the effect of sources, as in $[44,50]$. Therefore, any momentum equation based on $\left(p_{i}, \Pi_{\|}^{e s t}\right)$ is, in principle, inferior to employing a two-pressure $\left(p_{i \perp}, p_{i / l}\right)$ set of equations that includes complete energy equations for $p_{i \perp}$ and $p_{i / /}$.

Sources are potentially important causes of divergence between $p_{\perp}$ and $p_{\|}$, i.e. are potentially a strong influence on $\Pi_{i / /}$. The main source of ions in the edge is ionization of neutrals which 
have recycled from the solid surfaces on which the ions recombined. The recycling neutral velocity distribution is generally quite anisotropic, and is governed by various factors such as the angle between $\mathbf{B}$ and the solid surface from which the neutrals recycled. Thus at the instant of creation of the ions it is likely that $p_{i, \perp}$ and $p_{i, \|}$ differ significantly and in a way for which no simple generalizations are possible. An appropriate way to address this aspect is to use a two-pressure $\left(p_{i \perp}, p_{i \|}\right)$ formulation, taking the source terms from a neutral Monte Carlo code such as EIRENE, and employing the specific detailed geometry of the solid structures at the edge.

There is one further matter to deal with in order to close the equations: as noted in Sec. 5, calculation of the perpendicular viscous stress $\vec{\Pi}_{i \perp, \nabla B+c e n t}$ requires knowledge of the averages $\left\langle\tilde{v}_{i \|} \bar{v}_{i \perp}^{2}\right\rangle$ and $\left\langle\tilde{v}_{i \|}^{3}\right\rangle$, i.e. $q_{i \|}^{\perp}$ and $q_{i \|}^{\|}$. The sum of the two averages, $\left\langle\tilde{v}_{i \|} \bar{v}_{i \perp}^{2}\right\rangle$ and $\left\langle\tilde{v}_{i \|}^{3}\right\rangle$, is equal to $\left\langle\tilde{v}_{i \|} \tilde{v}_{i}^{2}\right\rangle$ which can be estimated - for strong collisionality - from the well known Spitzer, etc. expression for the heat flux: $q_{i \|} \equiv n m_{i}\left\langle\tilde{v}_{i \|} \tilde{v}_{i}^{2}\right\rangle / 2=-\chi_{i \|} \nabla_{\|} T_{i}$, with $\chi_{\|}=3.9 \frac{n T_{i} \tau_{i}}{m_{i}}$. Since, however, in Eq. (35) they are present with different weightings, they have to be calculated separately. For purposes of a rough estimate, however, we may simply take $2 q_{i \|}^{\|}+q_{i \|}^{\perp} \approx q_{i \|}$ to give: $\vec{\Pi}_{i \perp, \nabla B+c e n t} \sim \frac{m_{i} q_{i \|}}{Z e B^{3}} \mathbf{B} \times \vec{\nabla} B$, where we have also neglected the (usually) small difference between the directions of the $\nabla \mathrm{B}$ and centrifugal drifts and expressed both terms through the direction of the former.

A more refined estimate can be obtained from Braginskii's analysis, see App. B (which is however then dependant on the validity of his small perturbation assumption, i.e. the assumption of strong collisionality): the ratios $\left\langle\tilde{v}_{i \|}^{3}\right\rangle /\left\langle\tilde{v}_{i \|} \tilde{v}_{i}^{2}\right\rangle$ and $\left\langle\tilde{v}_{i \|} v_{i \perp}^{2}\right\rangle /\left\langle\tilde{v}_{i \|} \tilde{v}_{i}^{2}\right\rangle$ are equal to $3 / 5$ and $2 / 5$, respectively which introduces a factor $8 / 5$ in front of the RHS of the above expression:

$$
\vec{\Pi}_{i \perp, \nabla B+c e n t} \approx \frac{8}{5} \frac{m_{i} q_{i \|}}{Z e B^{3}} \mathbf{B} \times \vec{\nabla} B
$$

We will use the estimate of Eq. (44) rather than the rough estimate in terms of $p_{i}$ obtained in Sec. 5, where $n \mathbf{V}_{i \perp, \nabla B+c e n t}=\frac{2 p_{i}}{Z e B R} \vec{e}_{\text {vert }}$.

An estimate for the relative importance of the viscous force associated with this term can now be made by comparing it with e.g. the part of the ion viscous force related to parallel gradient of the ion parallel velocity $n T_{i} \tau_{i} V_{i \|} / L_{\|}^{2}$. The divergence $\vec{\nabla} \cdot \vec{\Pi}_{i \perp, \nabla B+c e n t}$ can be estimated as $\vec{\Pi}_{i \perp, \nabla B+c e n t} / \lambda_{S O L} \cdot \quad$ Using $q_{i \|} \sim \frac{n T_{i} \tau_{i}}{m_{i}} \nabla_{\|} T_{i}, \quad \mathbf{B} \times \vec{\nabla} B \sim B^{2} / R$ and making maximum estimates for the ion temperature gradient $\nabla_{\|} T_{i} \sim T_{i} / q R$, and for the viscous term 
$\nabla_{\|} \Pi_{i \|} \sim n T_{i} \tau_{i} v_{i, t h} /(q R)^{2}$, one can obtain: $\frac{\vec{\nabla} \cdot \vec{\Pi}_{i \perp, \nabla B+c e n t}}{\nabla_{\|} \Pi_{i \|}} \sim \frac{q \rho_{i}}{\Delta_{S O L}}=\frac{r}{R} \times \frac{\rho_{i \theta}}{\Delta_{S O L}}$. Therefore, this viscous term doesn't appear to be a dominant viscous contribution to the parallel momentum balance equation. We note, however, that we used here the maximum possible estimate for $\nabla_{\|} \Pi_{i \|}$, by assuming variation of the parallel ion velocity of order ion thermal velocity over the distance $q R$, which is unlikely to be realized in the main SOL plasma. At the same time, the estimate for the $\vec{\nabla} \cdot \vec{\Pi}_{i \perp, \nabla B+c e n t}$ term, which used $\nabla_{\|} T_{i} \sim T_{i} / q R$, is fairly realistic. This viscous term may therefore become quite an important contribution under certain plasma conditions.

It is computationally convenient to use the $\left(p_{\mathrm{i}}, \Pi_{\|}^{\text {est }}\right.$ ) (with the latter being an estimate for the parallel viscosity, for example one that assumes strong collisionality) form of the momentum equation rather than the $\left(p_{i \perp}, p_{i \|}\right)$ form with two complete energy equations. For conditions other than collision-dominated, however, this results in two deficiencies: (a) the values of $\Pi_{\|}^{e s t}$ are of uncertain validity, and (b) a false dependence on Larmor rotation is introduced to the momentum equation. Ongoing progress toward achievement of fusion power in devices such as tokamaks is resulting in hotter, less collisional boundary plasmas, motivating better approximations for the momentum equation based on two pressures - and employing complete energy equations for $p_{i \perp}$ and $p_{i \|}$.

This completes the process of closing the set of particle and momentum equations.

\section{Summary}

The system of particle and parallel momentum balance equations for plasma edge modelling derived in this paper is presented by Eq. (36), followed by expressions for the individual terms. For the most typical case of well Maxwellianized electrons, electron pressure is isotropic, so one can neglect the difference $\left(p_{e \|}-p_{e \perp}\right)$ and the total pressure anisotropy is due entirely to the ion component. While keeping parallel and perpendicular ion pressure as separate parameters represents a more general approach, a complete formulation then requires that the (two) energy equations be considered, a subject that we defer to a follow-on study. Therefore in the rest of this paper we will follow the standard approach and replace $\nabla_{\|} p_{i \|}$ with $\nabla_{\|}\left(p_{i}+\Pi_{i \|}\right)$, therefore formally introducing parallel ion viscous stress $\Pi_{i \|}$ into the equations. This is also necessary in order for us to be able to make direct comparisons with earlier formulations in the literature. The system Eq. (36) can then be formulated as follows:

$$
\left\{\begin{aligned}
\frac{\partial n}{\partial t}+\vec{\nabla} \cdot\left(n \mathbf{V}_{i \|}+n \mathbf{V}_{i \perp, d r}+n \mathbf{V}_{i \perp, \text { anom }}\right)=S & \frac{\partial\left(n m_{i} V_{i \|}\right)}{\partial t}+ \\
& \nabla_{\|}\left(p_{e}+p_{i}+n m_{i} V_{i \|}^{2}\right)-\left(n m_{i} V_{i \|}^{2}+p_{i \|}-p_{i \perp}\right) \nabla_{\|} B / B+ \\
& \vec{\nabla} \cdot\left(n m_{i} V_{i \|}\left(\mathbf{V}_{i \perp, d r}+\mathbf{V}_{i \perp, \text { anom }}\right)\right)+\vec{\nabla} \cdot\left(n m_{i} V_{i \|} \mathbf{V}_{i \perp, p \|}^{*}\right)+ \\
& \nabla_{\|} \Pi_{i \|}+\vec{\nabla} \cdot \vec{\Pi}_{i \perp, \nabla B+c e n t}+\vec{\nabla} \cdot \vec{\Pi}_{i \perp, \text { anom }}-n m_{i} V_{\|} \mathbf{V}_{E \times B} \cdot \mathbf{k}=F_{\|}
\end{aligned}\right.
$$


Expressions for the averaged ion drift flux $n \mathbf{V}_{i \perp, d r}$ and extra flux $n \mathbf{V}_{i \perp, p \|}^{*}$ which is only present in the momentum balance equation, are given by Eqs. (30) and (34), respectively, while 'anom' stands for anomalous contributions originating by e.g. fluctuating electromagnetic fields. Pressures $p_{e}$ and $p_{i}$ are full electron and ion pressures, respectively. The ion pressure anisotropy figures in the $3^{\text {rd }}$ and $6^{\text {th }}$ terms on the LHS of the momentum balance equation Eq. (45). The pressure difference $\left(p_{i \|}-p_{i \perp}\right)$ in the $3^{\text {rd }}$ term can be expressed as $3 \Pi_{i \|} / 2$ through the ion parallel viscous stress $\Pi_{i \|}$ given by Eq. (43) (while the parallel ion pressure itself, needed to calculate $n V_{i \|} \mathbf{V}_{i \perp, p \|}^{*}$, is given by $p_{i \|}=p_{i}+\Pi_{i \|}$ through the total ion pressure). The gradient $\nabla_{\|} \Pi_{i \|}$ can also be replaced with $2 \nabla_{\|}\left(p_{i \|}-p_{i \perp}\right) / 3$, where $p_{i \|}$ and $p_{i \perp}$ are considered independent quantities but this would then require two separate energy conservation equations for ion parallel and perpendicular pressures, as noted earlier.

The term $\vec{\nabla} \cdot \vec{\Pi}_{i \perp, \nabla B+c e n t}$ describes a force (taken with the opposite sign) that originates from viscous effects involving ion $\nabla \mathrm{B}$ and centrifugal drifts related to the deviation of the ion distribution function from the drifting Maxwellian, with the corresponding ion viscous stress given by Eq. (35). It can typically be simplified (see Eq. (44)). Anomalous viscous force is introduced separately by the term $\vec{\nabla} \cdot \vec{\Pi}_{i \perp, \text { anom }}$ which, together with the perpendicular particle anomalous flux $n \mathbf{V}_{i \perp, \text { anom }}$, is usually defined arbitrarily using a prescribed coefficient (see Sec. 5). The last term on the LHS of the momentum balance equation Eq. (45) accounts for the main part of the 'curvature force' for ions $F_{i, \text { curv }}=n m_{i}\left\langle v_{i \|} \mathbf{v}_{i \perp}\right\rangle \cdot \mathbf{k}$ (k is curvature of magnetic field lines) which was derived in Sec. 2 and for which approximate expressions are given by Eq. (29) (without specifying the charged particle species). Finally, $S$ and $F_{\|}$stand for particle source and all external forces acting on the plasma, including forces arising from interaction with neutrals. Perpendicular particle fluxes and forces arising due to Coulomb collisions have not been considered in the present paper due to their secondary importance. They can however be added separately to the equations, e.g. from Braginskii's analysis.

In deriving the above equations, effort has been made to include the most important terms and to explain their physical meaning. The importance of the terms is assessed by making order of magnitude estimates that take into account characteristic velocities, fluxes etc. present at the plasma edge, in particular in the SOL region of tokamaks. This system of equations, or its simplified (reduced) versions can be used in analytical theories and as well in sophisticated numerical codes.

One of the main motivations in deriving the present system of equations was the desire to clearly separate terms that have different origins, in particular: (a) guiding centre parallel and perpendicular (drift) velocities in quasi-stationary electro-magnetic fields and associated fluxes of parallel momentum, including viscous guiding centre drift effects, (b) perpendicular (radial) guiding centre anomalous fluxes and associated anomalous convective and viscous momentum fluxes, (c) pressure-related terms and parallel viscosity in the parallel momentum balance equations. Perpendicular classical diffusive fluxes originating from the distortion of Larmor orbits by the Coulomb collisions have not been included in the set of equations, however, they can in principal be added separately when these fluxes become particularly large. 
More detailed analysis of the parallel component of the gradient of the total pressure-stress tensor has identified a number of important terms that are sometimes not included in the set of particle and momentum balance equations for the edge plasma. One of them is the term $-\left(n m V_{\|}^{2}+p_{\|}-p_{\perp}\right) \nabla_{\|} B / B$ due to the spatial divergence of the magnetic field lines, while the other: $-n m\left\langle v_{\|} \mathbf{v}_{\perp}\right\rangle \cdot \mathbf{k}$, is due to the bending of the field lines described by their curvature k. At the same time, more detailed analysis of convective and viscous terms in the parallel momentum balance equation has led to the appearance of an extra term in the parallel momentum balance equation required to correctly account for the momentum transport by the ion centrifugal drift, namely, a significant contribution by the ion centrifugal drift to the viscous term $\vec{\nabla} \cdot\left(n m_{i}\left\langle\tilde{v}_{i \|} \widetilde{\mathbf{v}}_{i \perp, d r}\right\rangle\right)$, comparable to the contribution of this drift to the convective transport term $\vec{\nabla} \cdot\left(n m_{i} V_{i \|} \mathbf{V}_{i \perp, d r}\right)$, has been identified. For the drifting Maxwellian ion distribution function, the new term can be described by the expression $\vec{\nabla} \cdot\left(n m_{i} V_{i \|} \mathbf{V}_{i \perp, p \|}^{*}\right)$, having similar form to ordinary convective terms, with the $n \mathbf{V}_{i \perp, p \|}^{*}$ flux given by Eq. (34). This term is twice as large as the contribution of the random part of the ion centrifugal drift (described by the term with $p_{\|}$in Eq. (30)) to the convective momentum transport term. In situations where the ion parallel convective energy flux $n m_{i} V_{i \|}^{2}$ can be neglected compared with the ion pressure, and the pressure is isotropic, the presence of the new term effectively doubles parallel momentum transport described by the combined convective term $\vec{\nabla} \cdot\left(n m_{i} V_{i \|} \mathbf{V}_{i \perp, \nabla B+c e n t}\right)$ due to the ion $\nabla \mathrm{B}$ and centrifugal drifts. In addition, an extra perpendicular viscous term $\vec{\nabla} \cdot \vec{\Pi}_{i \perp, \nabla B+c e n t}$ for a general ion distribution function (not necessarily drifting Maxwellian, for which this term is zero), originating from the ion $\nabla \mathrm{B}$ and centrifugal drifts, with the vector $\vec{\Pi}_{i \perp, \nabla B+c e n t}$ given by Eqs. (35) (more precise form) and (44) (more approximate form), must be taken into account. Such a significant contribution of the ion centrifugal drift to the parallel momentum balance equation ultimately stems from the fact that the centrifugal drift velocity is proportional to $v_{i \|}^{2}$, thereby causing strongly nonlinear, $\sim v_{i \|}^{3}$, contributions of this drift to the parallel momentum balance equation.

The terms mentioned above are, as was shown earlier (see discussion following Eq. (24)), generally of the same order of magnitude as the convective momentum transport term attributed to drifts, $\vec{\nabla} \cdot\left(n m_{i} V_{i \|} \mathbf{V}_{i \perp, d r}\right)$, hence their inclusion in the parallel momentum balance equation is equally important as the inclusion of this straightforward convective term. There are a number of similarities between the present results and the results of an earlier study [35] - that is, over and above the inclusion of obvious pressure gradient, anomalous viscosity and convective momentum terms. First, the effect of roughly doubling of the convective momentum flux $\vec{\nabla} \cdot\left(n m_{i} V_{i \|} \mathbf{V}_{i \perp, d r}\right)$ attributable to the combination of the ion $\nabla \mathrm{B}$ and centrifugal drifts, due to the presence of the term $\vec{\nabla} \cdot\left(n m_{i} V_{i \|} \mathbf{V}_{i \perp, p \|}^{*}\right)$. Second, both formulations effectively contain what is here referred to as the 'curvature force', with its main part being $n m_{i} V_{\|} \mathbf{V}_{E \times B} \cdot \mathbf{k}$ : in [35], this contribution is contained as part of the term dubbed the 'Coriolis force' $F_{k}$ (see Eqs. (7) and (8) of [35] (although calling it 'Coriolis' is confusing since the true Coriolis force differs by a factor of 2)). The term 
$-\left(n m_{i} V_{i \|}^{2}+p_{i \|}-p_{i \perp}\right) \nabla_{\|} B / B$, however, was neglected in [35] as small. Also not included in [35] is the viscous term $\vec{\nabla} \cdot \vec{\Pi}_{i \perp, \nabla B+c e n t}$, as the analysis there implicitly assumes the ion distribution function to be a drifting Maxwellian. Finally, in this paper we presented a more complete expression for parallel viscosity, Eq. (43) [51], by including recent additions (note however, that viscous terms of [35] (contained inside the Eq. (7) of the Ref.) are also lacking radial (' $y$ ', according to the nomenclature adopted in [35]) gradients, an omission).

\section{Acknowledgements}

The authors gratefully acknowledge Drs. Coster D.P., Scott B., Simakov A. N., Smolyakov A., Profs. Lackner K. and Rozhansky V.A., for discussion of various aspects of the present paper. The authors particularly thank Dr. Mike Schaffer for a number of valuable insights and suggestions. PCS acknowledges the support of a Collaborative Research Opportunities Grant from the Natural Sciences and Engineering Research Council of Canada and by General Atomics and the Lawrence Livermore National Laboratory.

\section{References}

[1] Stangeby P C, in The Boundary of Magnetic Fusion Devices, IOP Publishing, Bristol (2000).

[2] Herrmann A, Junker W, Günther K, et al., Plasma Phys. Control. Fusion 37 (1995) 17.

[3] Asakura N, Itami K, Hosogane N et al., J. Nucl. Mater. 220-222 (1995) 395.

[4] Hutchinson I H, LaBombard B, Goetz J A et al., Plasma Phys. Control. Fusion 37 (1995) 1389.

[5] Chankin A V, J. Nucl. Mater. 241-243 (1997) 199.

[6] Feng Y, Herre G, Grigull P, Sardei F et al., Plasma Phys. Control. Fusion 40 (1998) 371.

[7] Boedo J A, Schaffer M J, Mainji R, Lasnier C J, Physics of Plasmas 7 (2000) 1075.

[8] Wenzel U, Coster D P, Kallenbach A, Kastelewicz H, Laux M, Maier H, Schneider R, et al., Nucl. Fusion 41 (2001) 1695.

[9] Lehnen M, Brix M, Samm U, Schweer B, Unterberg B et al., Nucl. Fusion 43 (2003) 168.

[10] Vershkov V A, Grashin S A, Chankin A V, J. Nucl. Mater, 145-147 (1987) 611.

[11] Pitts R A, Vayakis G, Matthews G F, and Vershkov V A, J. Nucl. Mater. 176 (1990) 843.

[12] Hugill J, J. Nucl. Mater. 196-198 (1992) 918.

[13] LaBombard B, Goetz J A, Hutchinson I, et al., J. Nucl. Mater. 241-243 (1997) 149.

[14] Asakura N, Sakurai S, Shimada M, Koide Y, Hosogane N, and Itami K, Phys. Rev. Lett. 84 (2000) 3093.

[15] Erents S K, Chankin A V, Mattews G F, Stangeby P C, Plasma Phys. Control. Fusion 42 (2000) 905.

[16] Coad J P, Bekris N and Elder J D et al., J. Nucl. Mater. 290-293 (2001) 224.

[17] Likonen J, Lehto S and Coad J P et al., Fus. Eng. Des. 66-68 (2003) 219.

[18] Matthews G F, J Nucl Mater 337-339 (2005) 1.

[19] Allen S L, et al, J Nucl Mater 337-339 (2005) 30.

[20] Elder J D, et al, J Nucl Mater 337-339 (2005) 79.

[21] McLean A G, et al, J Nucl Mater 337-339 (2005) 124.

[22] Gerhauser H and Claassen H A, J Nucl. Mater. 176\&177 (1990) 721. 
[23] Tendler M, Rozhansky V, Comments Plasma Phys. Control. Fusion 13 (1990) 191.

[24] Cohen R H, Ryutov D D, Phys. Plasma 2 (1995) 2011.

[25] Cohen R H, Ryutov D D, Comments Plasma Phys. Control. Fusion 16 (1995) 255.

[26] Radford G J, Chankin A V, Corrigan G, Simonini R, Spence J, Taroni A, Contrib. Plasma Phys. 36 (1996) 2/3, 187.

[27] Staebler G M, Nucl. Fusion 36 (1996) 1437.

[28] Schaffer M J, Chankin A V, Guo H Y, Matthews G F, Monk R D, Nucl. Fusion 37 (1997) 83.

[29] Zagorski R, Gerhauser H, Claaßen H A, Contrib. Plasma Phys. 38 (1998) Number 1-2, p. 61).

[30] Rognlien T D, Ryutov D D, Mattor N, Czech J. Phys. 48 (S2) (1998) 201.

[31] Rognlien T D, Ryutov D D, Mattor N, Porter G D, Phys. of Plasmas 6 (1999) 1851.

[32] Rognlien T D, Porter G D, Ryutov D D, J. Nucl. Mater 266-269 (1999) 654.

[33] Bonnin X, Rowan W L, Nucl. Fusion 39 (1999) 1009.

[34] Schneider R, Coster D, Braams B, et al., Contrib. Plasma Phys. 40 (2000) 328.

[35] Rozhansky V A, Voskoboynikov S P, Kaveeva E G, Coster D P, Schneider R, Nucl. Fusion 41 (2001) 387.

[36] Chankin A V, Corrigan G, Erents S K, Matthews G F, Spence J, Stangeby P C, J. Nucl. Mater. 290-293 (2001) 518.

[37] Porter G D, Rognlien T D, Rensink M E, Loarte A, Asakura N, Takenaga H, Matthews G, J. Nucl. Mater. 313-316 (2003) 1085.

[38] Coster D P, Bonnin X, Braams B, Reiter D, Schneider R, et al., Physica Scripta T108 (2004) 7.

[39] Erents S K, Pitts R A, Fundamenski W, Gunn J P and Matthews G F, Plasma Phys. Control. Fusion 46 (2004) 1757.

[40] Braginskii S I, in Reviews of Plasma Physics, edited by M.A.Leontovich (Consultants Bureau, New York, 1965), Vol. 1, p. 205.

[41] Mikhailovskii A B, Tsypin V S, Zh. Eksp. Teor. Fiz. 83 (1982) 139.

[42] Stangeby P C, Chankin A V, paper in preparation.

[43] Hinton F L, Hazeltine R D, Rev. Mod. Phys. 48 (1976) 239.

[44] Zawaideh E, Najamabadi F, and Conn R W, Phys. Fluids 29 (1986) 463.

[45] Zakharov L E and Shafranov V D, in Reviews of Plasma Physics, edited by M.A.Leontovich (Consultants Bureau, New-York, 1986), Vol. 11, p. 153.

[46] Sivukhin D V, in Reviews of Plasma Physics, edited by M.A.Leontovich (Consultants Bureau, New York, 1965), Vol. 1, p. 1.

[47] Hirshman S P, Sigmar D J, Nucl. Fusion 21 (1981) 1079.

[48] Hidalgo C, Gonçalves B, Silva C, Pedrosa M A, Erents K, Hron M, and Matthews G F, Plys. Rev. Letters 91 (2003) 065001.

[49] Radford G J, Contrib. Plasma Phys. 32 (1992) 297.

[50] Fundamenski W, Plasma Phys. Control. Fusion 47 (2005) R163-R208.

[51] Catto P J and Simakov A N, Physics of Plasmas 11 (2004) 90. 


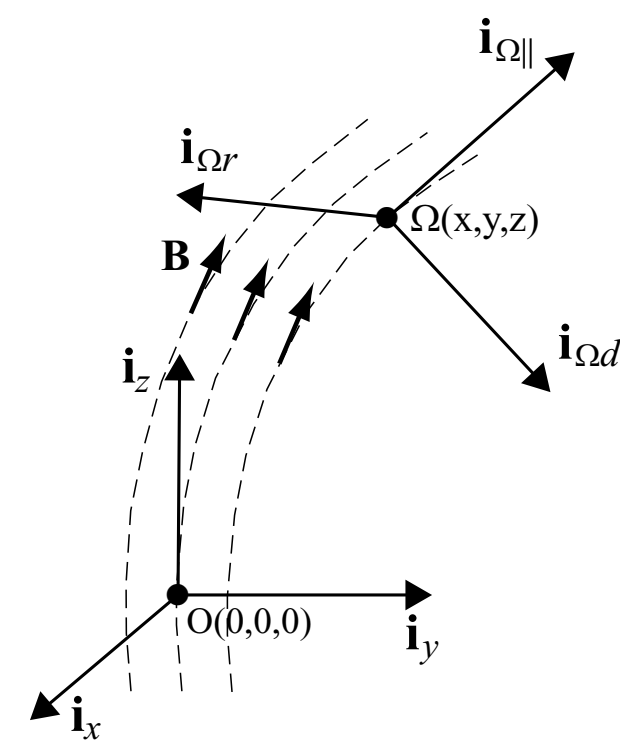

Fig. 1. Cartesian $\left(\mathbf{i}_{\mathbf{x}}, \mathbf{i}_{\mathbf{y}}, \mathbf{i}_{\mathbf{z}}\right)$ and local $\left(\mathbf{i}_{\Omega r}, \mathbf{i}_{\Omega d}, \mathbf{i}_{\Omega\|\|}\right)$ orthogonal systems of coordinates used for opening the parallel component of the gradient of the stress tensor, $(\vec{\nabla} \cdot \overrightarrow{\mathbf{P}})_{\|}$. 


\section{Appendix A: Estimates of the magnitude of drifts in the tokamak SOL}

In the simple SOL (sheath-limited) case, characteristic of low density operation, $T_{e}$ is constant (along B) right up to the target surface, $E_{r}$ is determined by the potential drop of $3 T_{e} / e$ in the Debye sheath between the plasma and the target [1]. Due to sharp radial gradients of $T_{e}$, a significant radial electric field $E_{r} \approx 3 T_{e} / e \lambda_{S O L}$ is formed in the SOL. In the other extreme case of very high plasma density, high recycling divertor conditions cause plasma detachment from the target, with $T_{e}$ decreasing strongly (along B) towards the target and another effect becomes largely responsible for the formation of the $E_{r}$, namely the parallel thermo-force $-0.71 n \partial T_{e} / \partial s_{\|}$(for singly charged ions, see e.g. [40]) that is nearly balanced by the parallel electric field force $e n E_{\|}$, resulting in $E_{r} \approx 0.71 \times T_{e} / e \lambda_{S O L}$, assuming absence of extremely high electron currents along the SOL. In either case the radial electric field in the SOL $E_{r} \sim T_{e} / e \lambda_{S O L}$. By assuming $T_{e}=T_{i}$, expressing ion temperature through ion thermal velocity as $T_{i} \sim m_{i} v_{i, t h}^{2}$, and introducing the ion Larmor radius as $\rho_{i}=v_{i, t h} / \omega_{i}$, where $\omega_{i}=e B / m_{i}$ is ion gyrofrequency, one can obtain the following estimate for poloidal $\mathrm{E} \times \mathrm{B}$ drift velocity in the SOL:

$V_{E \times B, \theta} \sim v_{i, t h} \times \rho_{i} / \lambda_{S O L}$

By replacing $m \bar{v}_{\|}^{2}$ and $m \bar{v}_{\perp}^{2}$ in the $4^{\text {th }}$ and $5^{\text {th }}$ terms on the RHS of Eq. (24) with $T_{i}$ and using similar derivations as above, one obtains for the $\nabla \mathrm{B}$ and centrifugal drift velocities, which are of the same order and which in the rest of the paper will be approximately denoted as $V_{\nabla B}$, the estimate:

$V_{\nabla B} \sim v_{i, t h} \times \rho_{i} / R$

The ratio of the two drift velocities, $V_{E \times B, p o l} / V_{\nabla B}$ is of order $R / \lambda_{S O L} \gg>1$. A similar (upper) estimate can be made for the radial component of the $\mathrm{E} \times \mathrm{B}$ drift, by allowing for a maximum potential variation of $\sim T_{e} / e$ along the flux surface creating a radial $\mathrm{E} \times \mathrm{B}$ drift velocity $V_{r} \sim T_{e} / e r B$. By assuming $T_{e} \sim m_{i} v_{i, t h}^{2}$, this velocity can be expressed as:

$V_{E \times B, r, \max } \sim v_{i, t h} \times \rho_{i} / r$

This drift velocity is much smaller than the typical poloidal ExB drift velocity, Eq. (A1), but is of the same order as $\nabla \mathrm{B}$ and centrifugal drift velocities, Eq. (A2). In some circumstances this allows one to neglect the $\nabla \mathrm{B}$ and centrifugal drifts compared with the $\mathrm{E} \times \mathrm{B}$ drift velocity which is predominantly in the poloidal direction (more correctly the direction perpendicular to $\mathbf{B}$ and within the flux surface, i.e. in the 'diamagnetic' direction - but in this paper we will often follow the usual custom and call this the 'poloidal' direction). However in some other circumstances $\nabla \mathrm{B}$ and centrifugal drifts, as well as radial component of the $\mathrm{E} \times \mathrm{B}$ drift, may have comparable effects to that of the poloidal $\mathrm{E} \times \mathrm{B}$ drift, when these drift velocities appear in the derivative expressions. Indeed, the large magnitude of the poloidal $\mathrm{E} \times \mathrm{B}$ drift is compensated by small changes, on the scale of minor radius $r$, in plasma density, resulting in 
the particle source/sink $\mathbf{V} \cdot \nabla n$ of order $V_{E \times B, \theta} n / r \sim n v_{i, t h} \times \rho_{i} / r \lambda_{S O L}$. At the same time, $\nabla \mathrm{B}$ and centrifugal drifts, being mostly in the vertical direction, have a large component across the field, i.e. perpendicular to the magnetic flux surface, i.e. in the 'radial' cross-field direction, and therefore may substantially influence the plasma through sharp radial gradients of $n_{e}, T_{e, i}$ at the edge. The correspondent particle source/sink is of order $V_{\nabla B} n / \lambda_{S O L} \sim n v_{i, t h} \times \rho_{i} / R \lambda_{S O L}$, which is only by a factor of the aspect ratio $R / r$ smaller than similar estimate for the poloidal $\mathrm{E} \times \mathrm{B}$ drift, while effect of the (maximum) radial electric field is the same as that of the poloidal $\mathrm{E} \times \mathrm{B}$ drift. In any case, the notion of the 'plasma size' used in the standard neoclassical theory, which figures in the denominator of the expression for $\delta$, becomes poorly defined for the SOL.

In addition to the above mentioned drift fluxes/velocities, there exist the diamagnetic flux which is equal to $n \mathbf{V}_{\text {dia }}=-\vec{\nabla} p_{\perp} \times \mathbf{B} /\left(Z e B^{2}\right)$. Its largest component is in the poloidal direction, $V_{d i a, \theta} \sim k T / e B \lambda_{S O L}$ and can be estimated similarly to an estimate Eq. (A1) given for the poloidal $\mathrm{E} \times \mathrm{B}$ drift. Its radial component can be given an upper estimate, similar to the estimate Eq. (A3) for the radial $\mathrm{E} \times \mathrm{B}$ drift velocity, by assuming large poloidal pressure gradients $\sim p / r$. This estimate, however, does not typically reflect the real experimental situation in the plasma, since plasma pressure, as well as electric potential, tend to be rather uniform along flux surfaces. The diamagnetic velocity, however, should not be called a 'drift' velocity, in the sense used in the present paper. We are reserving this term to mean the drift of the guiding centres of Larmor circles. It can be shown that the major part of the diamagnetic flux is due to the superposition of Larmor rotations which is given by $n \mathbf{V}_{\perp, L}=\operatorname{curl} \mathbf{M} / \mathrm{Ze}$, where $\mathbf{M}=-p_{\perp} \mathbf{B} / B^{2}$ is the magnetization flux [40,5]. The normalized difference between $n \mathbf{V}_{\text {dia }}$ and $\operatorname{curl} \mathbf{M} / Z$ Ze can be shown to be small, of order $\lambda_{S O L} / R$, the ion pressure decay length divided by major radius, i.e. the difference between $n \mathbf{V}_{\text {dia }}$ and $c u r l \mathbf{M} / \mathrm{Ze}$ is of order $V_{\nabla B} \quad$ (see e.g. $\left.[40,5]\right)$.

Another major difference with the core plasma, as has already been pointed out in the Introduction, is the possibility of large ion parallel velocity in the SOL, especially near the target (or limiter), where it reaches of order the plasma sound speed $c_{s}=\sqrt{\left(T_{e}+T_{i}\right) / m_{i}}$ due to the strong sink action of the target surface. Hence, the equations will include some parallel velocity terms which are neglected in the standard neoclassical theory, developed for configurations with closed field lines, on the basis of the $\delta$ - ordering. If one considers flows driven by ionization sources only then the near target region in the divertor with $v_{i \|} \sim c_{s}$ may occupy a relatively small volume of the plasma and one might expect that in the 'main SOL' (upstream of the entrance to the divertor) $v_{i \|}<<c_{s}$. However, even in the main SOL region, far away from the divertor/target, there is a possibility of a strong parallel flow in the SOL which is driven by - and can compensate for - the effect of the poloidal E $\times \mathrm{B}$ drift (a 'return parallel flow' considered in [23] and also found in the modelling with EDGE2D [36] and B2.5 [35] codes. The complete compensation of the poloidal $\mathrm{E} \times \mathrm{B}$ drift is achieved when the poloidal projection of the parallel velocity of the return parallel flow $\frac{B_{\theta}}{B} V_{E, \|}$ compensates 
the drift velocity $\frac{B_{\phi}}{B} \frac{E_{r}}{B}$, i.e. approximately $V_{E, \|}=\frac{E_{r}}{B_{\theta}}$. This flow is in the direction of the main plasma current. By making a similar estimate as above, one can obtain:

$$
V_{E, \|}=\frac{E_{r}}{B_{\theta}} \sim v_{i, t h} \times \frac{B}{B_{\theta}} \times \frac{\rho_{i}}{\lambda_{S O L}}=v_{i, t h} \times \frac{\rho_{i \theta}}{\lambda_{S O L}}
$$

where $\rho_{i \theta}$ is ion poloidal Larmor radius. This substantial velocity is typically below the ion sound velocity $c_{s}$, but may approach it in extreme cases. Such a return parallel flow also exists in the private region. Due to shorter parallel connection length there and, hence, shorter radial decay length (shorter than $\lambda_{S O L}$ ), the parallel velocity can even be larger in the private region than in the SOL.

The foregoing return parallel flow can occur in either cylindrical or toroidal configurations. In toroidal configurations, a different contribution to the parallel return flow occurs - the socalled Pfirsch-Schlüter flow, attributable to the existence of both radial electric field and ion pressure gradient in the SOL, has been previously considered [12]. Due to surface area variation $(\sim R)$ and variation in the magnetic field $(\sim 1 / R)$, the combined $\mathrm{E} \times \mathrm{B}$ and ion diamagnetic poloidal flow is significantly non-divergence-free, resulting in a compensating parallel flow which in a simple circular geometry with nested concentric surfaces, and assuming $\varepsilon=r / R<<1$, is given by $V_{P S, \|}=-\sin \theta \frac{2 q}{e n B}\left(\frac{d p_{i}}{d r}-e n E_{r}\right)$, where $q$ is the safety factor and $\theta$ poloidal angle measured from the bottom, see p. 561 of [1]. By replacing $d p_{i} / d r$ with $n T_{i} / \lambda_{S O L}$ and making other order of magnitude estimates as above, one obtains for the velocity of the ion Pfirsch-Schlüter parallel flow:

$V_{P S, \|} \sim v_{i, t h} \times \frac{q \rho_{i}}{\lambda_{S O L}}=v_{i, t h} \times \frac{r}{R} \times \frac{\rho_{i \theta}}{\lambda_{S O L}}$

This velocity is larger than the poloidal $\mathrm{E} \times \mathrm{B}$ drift velocity by a factor of $q$, but smaller than the velocity of the (possible) 'return parallel flow' mentioned earlier, Eq. (A4), by a factor $1 / \varepsilon$ (however, since it contains two contributions, one due to the radial electric field, and the other due to radial pressure gradient, numerically these two parallel flows can be close).

It is thus clear that quite fast parallel flow, driven by cross-field drifts, can occur in the SOL including locations far from the targets.

Appendix B: Calculation of averages $\left\langle\tilde{v}_{i \|} v_{i \perp}^{2}\right\rangle$ and $\left\langle\tilde{v}_{i \|}^{3}\right\rangle$

Braginskii's strong collisionality, perturbation analysis, which evaluates the departure of the velocity distribution from Maxwellian, shows, [40] (Eq. (4.17)), that the ion distribution function in the presence of parallel temperature gradient can be expressed in the form:

$$
f_{i}=f_{i}^{M}\left\{1+A\left(v^{2}\right) v_{\|}\right\}
$$


where $f_{i}^{M}$ is a non-shifted Maxwellian distribution function, $v$ and $v_{\|}$are particle full and parallel velocities, respectively, and the term with $A\left(v^{2}\right) v_{\|}$describes a small correction to $f_{i}^{M}: A\left(v^{2}\right) v_{\|}<<1$. In order to calculate parallel ion heat flux, the function $A\left(v^{2}\right)$ must satisfy the criterion: $V_{\|}=\frac{1}{n} \int f_{i} v_{\|} d \mathbf{v}=\frac{1}{n} \int f_{i}^{M} A\left(v^{2}\right) v_{\|}^{2} d \mathbf{v}=0$ (i.e., giving a zero net parallel ion flow).

Parallel ion heat flux can then be written as:

$q_{\|}=\int f_{i} \frac{m_{i} v^{2}}{2} v_{\|} d \mathbf{v}=\int f_{i}^{M} A\left(v^{2}\right) \frac{m_{i} v^{2}}{2} v_{\|}^{2} d \mathbf{v}$

The solution for the heat flux Eq. (B2) is well known from the literature and can, for example, be taken from Braginskii. Using the notations adopted in the main text of the present paper, this heat flux can be equated to $n m_{i}\left\langle\tilde{v}_{i \|} \tilde{v}_{i}^{2}\right\rangle / 2$, since the $v$ 's in the above equations are random velocities. For the purpose of calculating $\left\langle\tilde{v}_{i \|}^{3}\right\rangle$ it will be sufficient to find the relation between the (newly defined) flux

$q_{\|}^{\|}=\int f_{i} \frac{m_{i} v_{\|}^{2}}{2} v_{\|} d \mathbf{v}=\int f_{i}^{M} A\left(v^{2}\right) \frac{m_{i} v_{\|}^{2}}{2} v_{\|}^{2} d \mathbf{v}$

and Eq. (B2). The calculation of the ratio of the fluxes $q_{\|}^{\|}$and $q_{\|}$is very simple, and it doesn't depend on any details of the Maxwellian distribution, apart from its dependence on the full velocity $v$. In the integration, the velocity space element can be taken as $d \mathbf{v}=[2 \pi v(\sin \alpha) v d \alpha] d v$, where $\alpha=\arccos \left(v_{\|} / v\right)$ (after the integration over $\alpha$, the velocity space volume becomes $\left.\int_{0}^{\pi}[2 \pi v(\sin \alpha) v d \alpha] d v=4 \pi v^{2} d v\right)$. Since $v_{\|}$can be represented as $v \cos \alpha$, the difference between Eqs. (B2) and (B3) caused by the presence of $v^{2}$ in the former and $v_{\|}^{2}$ in the latter, can be reduced to different integrations over $\alpha$, whereas within each velocity space volume $4 \pi v^{2} d v$ the ratios between the integrals over $\alpha$ are the same. Integration over $v$ is therefore not required for the purpose of finding the ratio $q_{\|}^{\|} / q_{\|}$.

Eq. (B2) contains the following integral over $\alpha$ : $\int_{0}^{\pi} \cos ^{2} \alpha \sin \alpha d \alpha=2 / 3$, while Eq. (B3) has: $\int_{0}^{\pi} \cos ^{4} \alpha \sin \alpha d \alpha=2 / 5$. Hence, $\quad\left\langle\tilde{v}_{i \|}^{3}\right\rangle /\left\langle\tilde{v}_{i \|} \tilde{v}_{i}^{2}\right\rangle=q_{\|}^{\|} / q_{\|}=3 / 5 . \quad$ Noting $\quad$ that $\left(\left\langle\tilde{v}_{i \|} v_{i \perp}^{2}\right\rangle+\left\langle\tilde{v}_{i \|}^{3}\right\rangle\right)=\left\langle\tilde{v}_{i \|} \tilde{v}_{i}^{2}\right\rangle$, leads to $\left\langle\tilde{v}_{i \|} v_{i \perp}^{2}\right\rangle /\left\langle\tilde{v}_{i \|} \tilde{v}_{i}^{2}\right\rangle=q_{\|}^{\perp} / q_{\|}=2 / 5$. 